\title{
A Convergent 3-D Vortex Method With Grid-Free Stretching
}

\author{
By J. Thomas Beale*
}

\begin{abstract}
We prove the convergence of a vortex method for three-dimensional, incompressible, inviscid flow without boundaries. This version differs from an earlier one whose convergence was shown in [4] in that the calculation does not depend explicitly on the arrangement of the vorticity elements in a Lagrangian frame. Thus, it could be used naturally in a more general context in which boundaries and viscosity are present. It is also shown that previous estimates for the velocity approximation can be improved by taking into account the fact that the integral kernel has average value zero. Implications for the design of the method are discussed.
\end{abstract}

1. Vortex methods are a means of simulating time-dependent, incompressible fluid flow, in which the flow is represented by a collection of vorticity elements. The configuration of the elements at a given time determines an approximate velocity field. The velocity, in turn, is used to compute new positions for the elements, and, in the case of three-dimensional flow, the vorticity is updated in a similar way. The result is a system of ordinary differential equations for particle paths followed by the centers of the elements and, depending on the version, their vorticities as well, This approach has several important advantages. Computational elements are needed only in regions with vorticity, and obvious sources of numerical diffusion are avoided. Although it is generally difficult to maintain stability in numerical methods for nonlinear problems without some dissipation, the vortex methods discussed here are nonlinearly stable. As a consequence, this class of methods seems especially appropriate for inviscid or slightly viscous flows with concentrated vorticity. The main purpose of this paper is to prove convergence of one version of the vortex method for three-dimensional flows. This method was suggested by $\mathrm{C}$. Anderson; its properties were discussed in [1].

Vortex methods have been used for two-dimensional flow for some time, but 3-D calculations are newer, and the methods are still under development. Recent simulations of inviscid phenomena have included aircraft-trailing vortices and turbulent spots [22], vortex breakdown [25], the merger of vortex rings [17], and the possible formation of singularities in an initially smooth configuration [11], [28]. See Leonard [22], [23] for a more complete description of applications to date. When boundaries are present, the effect of viscosity must be incorporated, and there is some choice in how this can be done. In a general method developed by Chorin and

Received January 14, 1985; revised May 28, 1985.

1980 Mathematics Subject Classification. Primary 65M15, 76C05.

*Partially supported by O. N. R. Contract No. N00014-76-C-0316 and N. S. F. Grant No. NSF-DMS84-08393.

(c1986 American Mathematical Society $0025-5718 / 86 \$ 1.00+\$ .25$ per page 
others [10], vorticity elements of a second kind, representing vortex sheets, are generated at the boundary to satisfy the no-slip condition, and a random component is added to the velocity of all the elements to simulate viscosity. For a general description of this combined method, see [12]. Applications have included instabilities in flow past a flat plate [10], and start-up flow past a cylinder at high Reynolds number [9]. An alternative is to use a mesh in the boundary layer; airfoil calculations were done in this way in [29]. There are a number of related methods in use, such as cloud-in-cell methods; see [22], [23].

The convergence of vortex methods has been rigorously established for versions in two or three dimensions for inviscid flow without boundaries [19], [4], [5]. Other theory [8], [13], [24] lends partial support to the full method with boundary layers. The first proof of the convergence of vortex methods was given by Hald [18], [19] who established second-order convergence for two-dimensional flow by reducing the argument to consistency and stability estimates. A. Majda and the author, following the outline of Hald's approach, showed that a three-dimensional version also converges and that high-order accuracy can be achieved [4], [5]. (Hereafter, [4], [5] are referred to as [I] and [II].) In [6] we developed high-order accurate versions of the method given by simple, explicit formulas and illustrated the theoretical predictions with tests of exact solutions in 2-D. Further improvements in the theory have been made by Cottet and Raviart [14], [15], [26] and Anderson and Greengard [1]. For a concise summary of the present state of the theory, see [7].

In three-dimensional flow, unlike the simpler 2-D case, the vorticity is stretched and contracted by the changing geometry of the flow, and the numerical method must of course take this process into account. In the method of [I], which is in one sense the simplest possible version, the current vorticity is calculated from a difference approximation to the Jacobian matrix of the Eulerian coordinates of the elements with respect to the Lagrangian ones (see [I], [1], [7]). The differences are on a rectangular grid covering the support of the initial vorticity. Thus, the calculation makes explicit reference to the Lagrangian configuration of the particles, and this information must be retained in the computation. In the present method, however, the vorticity stretching term $(\omega \cdot \nabla) u$ is computed directly from the integral expression for the velocity (see (1.11) below), and the Lagrangian grid is not needed. In principle, then, this method could be combined with the vortex-sheet representation of the boundary layer, together with a random walk to simulate viscosity, as already described. This method was described in [1], and comparisons were made with the method of [I]. C. Anderson has also developed related methods for fluids of variable density using the Boussinesq approximation [2], [3].

The crucial stability estimate in the convergence argument assesses the error in the calculated velocity due to the error in the particle locations and in the transported values of vorticity. A similar estimate must be made for the errors in the calculated vorticities of the elements. For the method of [I] this second estimate followed more or less automatically from the first (see [I] or [1]), and it appeared that the stability might be a property special to this version of the method. However, the necessary estimates can be established for the present version through more detailed analysis, as shown in the last section of this paper. One implication is that more flexibility is allowed in the design of 3-D vortex methods, without loss of stability, than was thought earlier. 
Cottet and Raviart [14], [15] made the important observation that the treatment of consistency in [II] could be greatly simplified by regarding the error in discretizing the velocity integral directly as a quadrature error in Lagrangian coordinates. We follow their approach here, and derive improved estimates by taking into account the fact that the kernel of the integral operator has average value zero. Because of cancellation, the discretization is actually more accurate than it appears. The same observation holds, of course, for the discretization of the velocity integral in the two-dimensional vortex method. We also discuss modification of the present method to achieve the same advantage in the computed expression for the vorticities of the computational elements. Numerical examples are given to illustrate the improvement in accuracy possible from such modification. The derivation of the improved estimates is given in a supplement at the end of this issue.

We now describe the formulation of the vortex method to be discussed. For incompressible flow in $\mathbf{R}^{3}$ the velocity $u(x, t)$ can be found from the vorticity $\omega(x, t)$ according to the Biot-Savart Law

$$
u(x, t)=-\frac{1}{4 \pi} \int \frac{x-x^{\prime}}{\left|x-x^{\prime}\right|^{3}} \times \omega\left(x^{\prime}, t\right) d x^{\prime},
$$

assuming velocity zero at infinity. This expression has the form

$$
u(x, t)=\int K\left(x-x^{\prime}\right) \omega\left(x^{\prime}, t\right) d x^{\prime}
$$

with $K$ the matrix-valued kernel,

$$
K(x, t)=-\frac{1}{4 \pi} \frac{x}{|x|^{3}} \times=\nabla G(x) \times,
$$

where $G(x)=1 /(4 \pi|x|)$ is the Green's function for $-\nabla^{2}$. To discretize, we replace the kernel with a smoothed version, in direct analogy with two-dimensional vortex methods; see [II], [7], [19]. Let $\varphi_{\delta}(x)=\delta^{-3} \varphi(x / \delta)$ be a smooth approximation to the delta function, the length $\delta$ being a scaling parameter. We replace $G$ above by $G_{\delta}=G * \varphi_{\delta}$ and then $K$ by $K_{\delta}=K * \varphi_{\delta}$, where $*$ denotes convolution,

$$
K_{\delta}(x)=\nabla G_{\delta}(x) \times=\frac{\partial G_{\delta}}{\delta|x|}(x) \frac{x}{|x|} \times .
$$

It can be seen that $K_{\delta}$ has the form

$$
K_{\delta}(x)=-\frac{1}{4 \pi} f(|x| / \delta) \frac{x}{|x|^{3}} \times,
$$

with $f$ a function determined by $\varphi ; f(\rho) \rightarrow 1$ as $\rho \rightarrow \infty$, and $f(\rho)=O\left(\rho^{3}\right)$ as $\rho \rightarrow 0$, so that $K_{\delta}$ is smooth. The choice of $K_{\delta}$ and the relation between $\varphi$ and $f$ is discussed further in Section 2.

Our strategy will be to follow the trajectories

$$
d x_{i} / d t=u\left(x_{i}, t\right)
$$

of a collection of particles $\left\{x_{i}(t)\right\}$, each representing a material region of volume $h^{3}$; we will choose the particle locations at the initial time to be centers of grid cubes of side $h$. We assume the vorticity is zero outside a bounded set and write $\omega_{i}(t)=$ $\omega\left(x_{i}, t\right)$. The discrete version of the velocity is then

$$
u(x, t) \sim \sum_{j} K_{\delta}\left(x-x_{j}\right) \omega_{j} h^{3} .
$$


We will approximate the particle trajectories and their vorticities by a system of ordinary differential equations, with the particle velocities computed from this discrete velocity field. The most familiar expression for the evolution of vorticity is

$$
\omega_{t}+(u \cdot \nabla) \omega=(\omega \cdot \nabla) u .
$$

Thus, to find the material derivative of $\omega$, we would like to form an approximation for $(\omega \cdot \nabla) u$ in terms of $\left\{x_{j}, \omega_{j}\right\}$. A natural way to evaluate $\nabla u$ is to differentiate (1.6), applying the gradient to the smooth kernel $K_{\delta}$ :

$$
\nabla u \sim \sum_{j} \nabla K_{\delta}\left(x-x_{j}\right) \omega_{j} h^{3} .
$$

More explicitly, we have

$$
\frac{\partial u_{k}}{\partial x_{l}} \sim \sum_{j} \sum_{m, n} \varepsilon_{k m n} G_{\delta, l m}\left(x-x_{j}\right)\left(\omega_{j}\right)_{n} h^{3},
$$

where $\varepsilon_{k m n}$ is the usual permutation tensor and indices after a comma denote derivatives. Applying (1.8) to (1.7), we write

$$
\frac{d \omega_{i}}{d t}=\frac{d}{d t} \omega\left(x_{i}(t), t\right) \sim \omega_{i} \cdot \sum_{j} \nabla K_{\delta}\left(x-x_{j}\right) \omega_{j} h^{3} .
$$

Finally, we obtain a system of differential equations for the particle locations $\left\{x_{i}(t)\right\}$ and their vorticities $\left\{\omega_{i}(t)\right\}$ approximating (1.5), (1.7):

$$
\begin{gathered}
\frac{d \tilde{x}_{i}}{d t}=\sum_{j} K_{\delta}\left(\tilde{x}_{i}-\tilde{x}_{j}\right) \tilde{\omega}_{j} h^{3}, \\
\frac{d \tilde{\omega}_{i}}{d t}=\tilde{\omega}_{i} \cdot \sum_{j} \nabla K_{\delta}\left(\tilde{x}_{i}-\tilde{x}_{j}\right) \tilde{\omega}_{j} h^{3} .
\end{gathered}
$$

(We use tildes to distinguish the solutions of the O.D.E.'s from the corresponding quantities in the actual flow.) Once $\left\{\tilde{x}_{i}, \tilde{\omega}_{i}\right\}$ are known, the velocity field can be computed from (1.6); a similar expression for the vorticity is discussed below. We prove here that all these quantities converge to the correct values as the particle spacing and the smoothing parameter approach zero in a certain way; see Section 2 for precise statements. An insightful discussion of this method is given in Section 4 of [1]. The method (1.10), (1.11) and that of [I] are closely related to the method of Chorin [10] and also similar in spirit to earlier calculations such as Leonard [21], [22]. Saffman [27] coined the term "vortons" for the elements in a discretization of (1.5), (1.7).

A variant of $(1.10),(1.11)$ is based on the familar fact that

$$
\nabla u \cdot \omega=\nabla^{s} u \cdot \omega
$$

where $\nabla^{s} u$ is the symmetric part of $\nabla u$, i.e., the rate-of-strain tensor

$$
\nabla^{s} u=\frac{1}{2}\left(\nabla u+(\nabla u)^{T}\right),
$$

with $T$ indicating the transpose. We might therefore replace (1.11) with

$$
\frac{d \tilde{\omega}_{i}}{d t}=\tilde{\omega}_{i} \cdot\left\{\sum_{j} \nabla K_{\delta}\left(\tilde{x}_{i}-\tilde{x}_{j}\right) \tilde{\omega}_{j} h^{3}\right\}^{s},
$$


$s$ meaning the symmetric part. Whereas (1.9) involves arbitrary second derivatives of $G_{\delta}$, the corresponding symmetrized version uses only mixed partial derivatives or a difference of two repeated derivatives: If $k=l$ in (1.9), repeated derivatives occur only for $m=l=k$ and the term in the sum is zero. If on the other hand $k \neq l$, $m=l$, there is a unique value of $n$ so that $\varepsilon_{k m n}=\varepsilon_{k l n}$ is nonzero. If $k$ and $l$ are switched we have the two terms

$$
\varepsilon_{k l n} G_{\delta, l l}\left(\omega_{j}\right)_{n}+\varepsilon_{l k n} G_{\delta, k k}\left(\omega_{j}\right)_{n}= \pm\left(G_{\delta, l l}-G_{\delta, k k}\right)\left(\omega_{j}\right)_{n}
$$

as claimed. As a result, the kernel whose discretization appears in (1.13) has average value zero. It is shown in Section 4 that this property can be used to modify (1.13) so that improved consistency estimates are possible for the discretization of $(\omega \cdot \nabla) u$, and numerical examples are given in Section 2 in which improvement is evident. On the other hand, Greengard has pointed out that (1.10), (1.11) has an advantage which is apparently not shared by the symmetrized version; namely, the computed flow map preserves vortex lines in a certain natural sense, as does the actual flow (see Section 4 of [1]). Thus, in a computation with only a few vortex lines, the alignment of vorticity arrows may appear more realistic in the first method.

The smoothing of the kernel followed by discretization can be interpreted as an approximation of the vorticity by a sum of elements of "blobs" of vorticity centered at the particle locations. The expression for the computed velocity field (1.6) is equivalent to

$$
\begin{aligned}
u(x, t) & \sim \sum_{j} \nabla G_{\delta}\left(x-x_{j}\right) \times \omega_{j} h^{3} \\
& =\nabla \times \sum_{j} G_{\delta}\left(x-x_{j}\right) \omega_{j} h^{3} \\
& =\nabla \times\left(-\nabla^{2}\right)^{-1} \sum_{j} \varphi_{\delta}\left(x-x_{j}\right) \omega_{j} h^{3} .
\end{aligned}
$$

A natural vorticity approximation is the curl of the above,

$$
\omega(x, t) \sim \nabla \times \nabla \times\left(-\nabla^{2}\right)^{-1} \sum_{j} \varphi_{\delta}\left(x-x_{j}\right) \omega_{j} h^{3} .
$$

This is, in fact, the orthogonal projection, in the $L^{2}$ sense, of the "blob" sum

$$
\sum_{j} \varphi_{\delta}\left(x-x_{j}\right) \omega_{j} h^{3} \equiv b(x, t)
$$

onto the subspace of divergence-free vector fields. Suppose that, conversely, we began with (1.14) as an approximation $\omega^{h}(x, t)$ to the vorticity, so that $\omega^{h}=P b, P$ being the projection; then $(I-P) b$ is a gradient, and consequently the velocity field corresponding to $\omega^{h}$ is, using (1.2),

$$
\nabla \times\left(-\nabla^{2}\right)^{-1} \omega^{h}=\nabla \times\left(-\nabla^{2}\right)^{-1} b=\sum_{j} \nabla G_{\delta}\left(x-x_{j}\right) \times \omega_{j} h^{3},
$$

which is the same as (1.6). Thus (1.14) is the representation of the vorticity as a collection of elements, as mentioned earlier. It determines the velocity field (1.6) used to update the location of the elements. We can view (1.14) as the analogue of the approximation of the scalar vorticity in two dimensions by a sum of "blobs" [7], [19]. 
The rest of this paper is organized as follows. In Section 2 we discuss the choice of the modified kernel $K_{\delta}$ and describe the convergence results in detail, as well as the consistency and stability lemmas. We also present a numerical example illustrating the discretization of the velocity $u$ and the stretching term $(\omega \cdot \nabla) u$. Section 3 gives the proof of convergence, assuming the consistency and stability lemmas, and reviews the simplified treatment of consistency. The stability argument is presented in Section 5, after a few lemmas in Section 4. Finally, the improved consistency estimates, taking into account the average-zero property of the kernel, are derived in the Supplement. (These improved estimates are not necessary for the proof of convergence.)

It is a pleasure to thank A. Majda for a number of stimulating conversations concerning the questions dealt with here. The author also wishes to thank Majda and A. Chorin for arranging a visit at the University of California at Berkeley during which this work was undertaken.

2. Summary of Results. We begin by introducing some notation. Let $\Lambda$ be the lattice of points in $\mathbf{R}^{3}$ with integer coordinates and $\Lambda^{h}$ the discrete grid $h \Lambda$ of size $h$. We assume the initial vorticity is zero outside some radius $R_{0} ; \Lambda_{0}^{h}$ will denote the intersection of $\Lambda^{h}$ with $\left\{x:|x|<R_{0}\right\}$. We write $\alpha$ for the Lagrangian position. The particle trajectory

$$
\frac{d x}{d t}=u(x, t), \quad x(0 ; \alpha)=\alpha,
$$

determines a coordinate mapping $\Phi^{t}(\alpha)=x(t ; \alpha)$ from Lagrangian to Eulerian location. We discretize the flow by introducing particles at time zero at locations $\alpha_{i}=i h, i h \in \Lambda_{0}^{h}$. Let $x_{i}(t)=\Phi^{t}\left(\alpha_{i}\right)$ and $\omega_{i}(t)=\omega\left(x_{i}(t), t\right)$. Associated with the particle configuration we have approximate velocity and vorticity fields, as in (1.6), (1.14),

$$
\begin{gathered}
u^{h}(x, t)=\sum_{j h \in \Lambda_{0}^{h}} K_{\delta}\left(x-x_{j}\right) \omega_{j} h^{3}, \\
\omega^{h}(x, t)=\sum_{j h \in \Lambda_{0}^{h}} \nabla \times\left[K_{\delta}\left(x-x_{j}\right) \omega_{j} h^{3}\right] .
\end{gathered}
$$

In a corresponding way, $\tilde{x}_{i}(t)$ and $\tilde{\omega}_{i}(t)$ will denote the solutions of the ordinary differential equations (1.10), (1.11) or (1.10), (1.13), the sums being over $j h \in \Lambda_{0}^{h}$. The initial data is the same as for the actual flow:

$$
\tilde{x}_{i}(0)=\alpha_{i}, \quad \tilde{\omega}_{i}(0)=\omega_{0}\left(\alpha_{i}\right) .
$$

Similarly, we write $\tilde{u}^{h}(x, t), \tilde{\omega}^{h}(x, t)$ for the expressions analogous to (2.2), (2.3) with $x_{j}, \omega_{j}$ replaced by $\tilde{x}_{j}, \tilde{\omega}_{j}$. We also write for simplicity

$$
\tilde{u}_{i}^{h}(t)=\tilde{u}^{h}\left(\tilde{x}_{i}(t), t\right), \quad u_{i}^{h}(t)=u^{h}\left(x_{i}(t), t\right),
$$

and similarly for $\nabla \tilde{u}_{i}^{h}, \nabla u_{i}^{h}$.

We now discuss the choice of the smooth delta function $\varphi_{\delta}$ and the modified velocity kernel $K_{\delta}$, where again

$$
\varphi_{\delta}(x)=\delta^{-3} \varphi(x / \delta), \quad K_{\delta}=K * \varphi_{\delta} .
$$


Our assumptions on $\varphi$ will be that

(i) $\varphi$ is a smooth, even function of the radius $r=|x|$, decaying at infinity;

(ii) $\int \varphi(x) d x=1$;

(iii) $\int x^{\beta} \varphi(x) d x=0$ for $1 \leqslant|\beta| \leqslant m-1$.

The decay at infinity will be made more specific below. Here $\beta$ is a multi-index and $m$ is a positive integer. Since (iii) holds by symmetry for $\beta$ odd, we can take $m$ to be even. In fact, (iii) is satisfied for any radial $\varphi$ with $m=2$, and higher order can be achieved by imposing further conditions. The value of $m$ determines the order of accuracy of the method in the convergence theorem.

If $\varphi$ is chosen, it is easily seen by an integration that the modified velocity kernel $K_{\delta}$ has the form (1.4), with

$$
\varphi(x)=\frac{f^{\prime}(r)}{4 \pi r^{2}}, \quad r=|x| .
$$

We may equivalently choose $\varphi$ or $f$, and it is natural to make a choice of $f$ so that $K_{\delta}$ has a simple expression. This approach was developed in [6]. It was shown that the conditions (i)-(iii) on $\varphi$ will hold, provided $f$ satisfies

(F1) $f(r) / r^{3}$ is a smooth function of $r^{2}$;

(F2) $f(r) \rightarrow 1$ as $r \rightarrow \infty$;

(F3) $\int_{0}^{\infty} f^{\prime}(r) r^{2 k} d r=0, \quad 2 \leqslant 2 k \leqslant m-2$;

and $f(r)-1$ decays well enough at infinity. Specifically, we will assume

(F4) $\left|D^{j} f(r)\right| \leqslant C_{j} r^{-l-j}, r \geqslant 1$,

for each $j \geqslant 1$ and a fixed $l$. It is sufficient for our results here to suppose $l \geqslant m+1$. With $l=m+1,(2.5)$ and (F4) imply that

$$
\left|D^{j} \varphi(r)\right| \leqslant C_{j} r^{-(m+4+j)} .
$$

It was noted in [6] that if $f_{2}$ is a function satisfying (F1), (F2), then

$$
f(r)=f_{2}(r)+\frac{1}{2} r f_{2}^{\prime}(r)
$$

satisfies (F1)-(F3) with $m=4$. Two choices which have worked reasonably well in preliminary tests are based on the second-order functions

$$
f_{2}(r)=\left(\tanh r^{2}\right)^{3 / 2}, \quad f_{2}(r)=r^{3} /\left(1+r^{6}\right)^{1 / 2} .
$$

The corresponding fourth-order versions are

$$
\begin{gathered}
f(r)=T^{3 / 2}+\frac{3}{2} r^{2} T^{1 / 2}\left(1-T^{2}\right), \quad T=\tanh r^{2}, \\
f(r)=r^{3}\left\{\left(1+r^{6}\right)^{-1 / 2}+\frac{3}{2}\left(1+r^{6}\right)^{-3 / 2}\right\} .
\end{gathered}
$$

These have performed somewhat better in preliminary tests than the two examples given in [6], perhaps because the earlier ones decay extremely rapidly. Some sample errors for computed values of $u$ and $(\omega \cdot \nabla) u$ at time zero are given below.

In stating the convergence results below we assume that the solution $u$ of the Euler equations is sufficiently smooth for the time interval under consideration. It is known that, if the initial data has several derivatives in $L^{2}$, then a classical solution exists for a time interval depending on a norm of the initial data, and the solution has the same number of derivatives in $L^{2}$ as the data. The time interval is independent of the number of derivatives. (See [20], [31] for precise statements and proofs.) Thus, the smoothness assumptions on the solution can be regarded as 
assumptions about the initial data. Regularity of the velocity field implies that the derivatives of the flow map $\Phi^{t}$ are bounded.

We will measure errors in continuous and discrete $L^{p}$-norms and related norms. These are most convenient for the stability estimates because of the central role of singular integral operators. The discrete $L^{p}$-norm for $u_{i}$ defined on $i h \in \Lambda^{h}$ is

$$
\left|u_{i}\right|_{0, p, h}=\left\{\sum_{i h \in \Lambda^{h}}\left|u_{i}\right|^{p} h^{3}\right\}^{1 / p} \text {. }
$$

We also use the discrete inner product

$$
\left(u_{i}, v_{i}\right)_{h}=\sum_{i h \in \Lambda^{h}} u_{i} v_{i} h^{3} .
$$

If the velocity is measured in $L_{h}$, it is natural to measure the vorticity in a discrete version of the negative Sobolev space $W^{-1, p}$. We first define the $W_{h}^{1, p}$-norm as

$$
\left|g_{i}\right|_{1, p, h}^{p}=\left|g_{i}\right|_{0, p, h}^{p}+\sum_{j=1}^{3}\left|D_{j}^{+} g_{i}\right|_{0, p, h}^{p},
$$

where $D_{j}^{+}$is the usual forward-difference operator in the $j$ th coordinate direction. As in the continuous case, we then define $W^{-1, p}$ as the dual space to $W^{1, p^{*}}$, $(1 / p)+\left(1 / p^{*}\right)=1$. Thus,

$$
\left|w_{i}\right|_{-1, p, h}=\sup _{\left\{g_{i}\right\} \in W_{h}^{1, p^{*}}} \frac{\left|\left(w_{i}, g_{i}\right)_{h}\right|}{\left|g_{i}\right|_{1, p^{*}, h}} .
$$

It follows easily that

$$
\begin{gathered}
\left|w_{i}\right|_{-1, p, h} \leqslant\left|w_{i}\right|_{0, p, h}, \\
\left|w_{i}\right|_{0, p, h} \leqslant 2 h^{-1}\left|w_{i}\right|_{-1, p, h}, \\
\left|D_{j}^{+} g_{i}\right|_{-1, p, h} \leqslant\left|g_{i}\right|_{0, p, h} .
\end{gathered}
$$

(For the last, see Proposition 2.1 in [I].) Finally, we are ready to state the main result.

Convergence Theorem. Assume that the velocity field $u(x, t)$ is smooth for $0 \leqslant t \leqslant T$ and that the initial vorticity $\omega_{0}(x)$ is zero outside a bounded set. Suppose that the modified kernel $K_{\delta}$ is chosen subject to conditions (F1)-(F4) with $m \geqslant 4$ and $l \geqslant m+1$. Set $\delta=c_{0} h^{q}$ for fixed $c_{0}$ and $q$ with $\frac{1}{3}<q<1$. Choose $p$ with $1<p<\infty$. Then, for $0 \leqslant t \leqslant T$, the following convergence estimates hold for the method defined by (1.10), (1.11), (2.4), or the symmetrized version with (1.11) replaced by (1.13):

(1) the particle paths

$$
\left|\tilde{x}_{i}(t)-x_{i}(t)\right|_{0, p, h} \leqslant C h^{m q},
$$

(2) the particle velocities

$$
\left|\tilde{u}_{i}^{h}(t)-u\left(x_{i}(t), t\right)\right|_{0, p, h} \leqslant C h^{m q},
$$

(3) the particle vorticities

$$
\left|\tilde{\omega}_{i}(t)-\omega\left(x_{i}(t), t\right)\right|_{-1, p, h} \leqslant C h^{m q},
$$


(4) the velocity field

$$
\left|\tilde{u}^{h}(\cdot, t)-u(\cdot, t)\right|_{L^{p}(B(R))} \leqslant C h^{m q},
$$

(5) the vorticity field

$$
\left|\tilde{\omega}^{h}(\cdot, t)-\omega(\cdot, t)\right|_{L^{p}(B(R))} \leqslant C h^{(m-1) q},
$$

where $B(R)=\{x:|x|<R\}$. The constants depend on $T, \varphi, q, c_{0}, R$, and bounds for several derivatives of $u$.

We were not able to include the case $m=2$ in our stability argument; this seems inconsequential, since there is no disadvantage in using fourth-order kernels. The difference between the two versions of the method does not appear in the statement of the theorem; it does appear, however, in the consistency estimates below. The inequality (3) for the particle vorticities can be converted to an estimate in $L_{h}^{p}$ using (2.10).

The following two lemmas are the essential parts of the convergence proof.

CONSISTENCY LEMMA. With the same hypothesis as the Convergence Theorem, except that $h$ and $\delta$ are regarded as independent parameters, we have

$$
\begin{gathered}
\left|u^{h}(x, t)-u(x, t)\right| \leqslant C\left(\delta^{m}+\delta(h / \delta)^{l}\right), \\
\left|\nabla u^{h}(x, t)-\nabla u(x, t)\right| \leqslant C\left(\delta^{m}+(h / \delta)^{l}\right), \\
\left|\omega^{h}(x, t)-\omega(x, t)\right| \leqslant C\left(\delta^{m}+(h / \delta)^{l}\right) .
\end{gathered}
$$

The estimates hold uniformly in $x$ and $t$ for $|x| \leqslant R$ and $0 \leqslant t \leqslant T$, where $R$ is an arbitrary radius. Here l can be taken arbitrarily large, depending on the smoothness of the solution, and the constants depend on $l$.

Stability Lemma. With the hypothesis of the Theorem, choose $\varepsilon$ and $p$ so that $0<\varepsilon<q / 2,2 \varepsilon<3 q-1$, and $p>3 / \varepsilon$. Suppose that for some time $t$ with $0 \leqslant t \leqslant T$ we have

$$
\eta(t) \equiv\left|\tilde{x}_{i}(t)-x_{1}(t)\right|_{0, p, h}+\left|\tilde{\omega}_{i}(t)-\omega_{i}(t)\right|_{-1, p, h} \leqslant h^{m q-\varepsilon} .
$$

Then the following estimates hold:

$$
\begin{gathered}
\left|\tilde{u}_{i}^{h}(t)-u_{i}^{h}(t)\right|_{0, p, h} \leqslant C \eta(t), \\
\left|\nabla \tilde{u}_{i}^{h}(t) \tilde{\omega}_{i}(t)-\nabla u_{i}^{h}(t) \omega_{i}(t)\right|_{-1, p, h} \leqslant C \eta(t), \\
\left|\tilde{u}^{h}(\cdot, t)-u^{h}(\cdot, t)\right|_{L^{p}(B(R))} \leqslant C \eta(t), \\
\left|\tilde{\omega}^{h}(\cdot, t)-\omega^{h}(\cdot, t)\right|_{L^{p}(B(R))} \leqslant C \delta^{-1} \eta(t) .
\end{gathered}
$$

The constants are independent of $t$. (S2) also holds with $\nabla u$ replaced by $\nabla^{s} u$.

The consistency estimates for the velocity and symmetrized gradient, evaluated at the particle locations, can be improved by taking into account the average value zero of the kernels. The following conclusions are described further in the Supplement.

IMPROVED CONSISTENCY LEMMA. With the hypothesis as in the Consistency Lemma, we have for $0 \leqslant t \leqslant T$,

$$
\left|u_{i}^{h}(t)-u\left(x_{i}(t), t\right)\right| \leqslant C\left(\delta^{m}+\delta^{2}(h / \delta)^{l}\right)
$$

At time zero, we have

$$
\left|\nabla^{s} u_{i}^{h}(0)-\nabla^{s} u\left(\alpha_{i}, 0\right)\right| \leqslant C\left(\delta^{m}+\delta(h / \delta)^{l}\right) .
$$


Moreover, $\nabla^{s} u_{i}^{h}$ can be replaced by a modified discretization $\nabla_{0}^{s} u_{i}^{h}$ of $\nabla^{s} u$ so that

$$
\left|\nabla_{0}^{s} u_{i}^{h}(t)-\nabla^{s} u\left(x_{i}(t), t\right)\right| \leqslant C\left(\delta^{m}+\delta(h / \delta)^{l}\right)
$$

for $0 \leqslant t \leqslant T$.

As mentioned before, the improved consistency estimates do not affect the statement of the Convergence Theorem, because we assume $l$ large enough so that the term $O\left(\delta^{m}\right)$ in the error dominates the rest. However, it will be apparent in the proof of the theorem that the total error estimate is improved by these more special consistency estimates if we regard $h$ and $\delta$ as independent parameters.

To illustrate the difference between the two expressions for vorticity stretching, we describe the relative errors in a calculation of $u$ and $\nabla u \cdot \omega$ at time zero for a simple test problem. With modest resolution, the errors in the unsymmetrized form of the stretching term were $20 \%-50 \%$ higher than those of the symmetrized version. We took the velocity field to be

$$
\left(1-r^{2}\right)^{4}\left(-x_{2}, x_{1}, 0\right), \quad r=|x| \leqslant 1,
$$

and zero otherwise, corresponding to the vorticity

$$
2\left(1-r^{2}\right)^{3}\left(4 x_{1} x_{3}, 4 x_{2} x_{3}, 1-5 x_{1}^{2}-5 x_{2}^{2}-x_{3}^{2}\right) .
$$

We covered the support with a cubic grid of 17 intervals across and assigned vorticities at the centers of the grid cubes. We computed $u, \nabla u \cdot \omega,(\nabla u)^{s} \cdot \omega$ according to (1.10), (1.11), (1.13) in the upper quadrant of the $x_{2} x_{3}$-plane. Because of the symmetry, these values should be typical. We use the fourth-order kernels (2.7), (2.8), and also a version of (2.8) which satisfies (F3) with $m=6$,

$$
f(r)=r^{3}\left\{\left(1+r^{6}\right)^{-1 / 2}+\frac{27}{8}\left(1+r^{6}\right)^{-5 / 2}\right\} .
$$

The relative $L^{2}$ errors are given in Table 1. For reference, the errors in computing $u$ or $(\nabla u)^{s} \cdot \omega$ with no smoothing are $3.6 \%$ and $2.6 \%$. The relative error was found as the square root of a ratio of integrals evaluated by the trapezoidal rule,

$$
\left(\sum_{i_{2}, i_{3} \geqslant 0} \sigma_{i}\left|u_{i}^{h}-u_{i}\right|^{2} \rho_{i} h^{2}\right) /\left(\sum_{i_{2}, i_{3} \geqslant 0} \sigma_{i}\left|u_{i}\right|^{2} \rho_{i} h^{2}\right),
$$

where $i=\left(0, i_{2}, i_{3}\right), \rho_{i}=h \mid i_{2}$, and $\sigma_{i}=.5$ if $i_{3}=0, \sigma_{i}=1$ otherwise. The factor of $\rho_{i}$ is inserted to account for rotational symmetry about the $x_{3}$-axis.

\section{TABLE 1}

Percentage errors for three modified kernels

\begin{tabular}{c|cccc|cccc|ccc}
\multicolumn{4}{c}{ Kernel (i) } & \multicolumn{4}{c}{ Kernel (ii) } & \multicolumn{3}{c}{ Kernel (iii) } \\
\hline$\delta$ & 1 & 1.5 & 2 & 2.2 & 1 & 1.5 & 2 & 2.3 & 1.5 & 2 & 2.5 \\
\hline$u$ & .48 & 1.3 & 4.0 & 5.5 & .67 & 1.2 & 4.2 & 6.4 & .69 & .94 & 2.1 \\
$\nabla u \cdot \omega$ & 2.8 & 3.1 & 6.4 & 9.2 & 4.6 & 4.0 & 6.6 & 10.4 & 2.9 & 2.7 & 1.9 \\
$(\nabla u)^{s} \cdot \omega$ & 2.3 & 2.5 & 4.8 & 6.8 & 3.8 & 3.2 & 4.9 & 7.8 & 2.0 & 1.9 & 1.3 \\
& & & & & & & & & & & \\
\hline
\end{tabular}

Errors obtained with kernels determined by (i) (2.7),

(ii) (2.8), and (iii)(2.13). 
3. Proofs of Convergence and Consistency. In this section we present the proof of the Convergence Theorem, using the lemmas of consistency and stability stated above. We then discuss the derivation of the consistency estimates, largely relying on previous work.

The theorem is easily proved by using the Consistency and Stability Lemmas to obtain a differential inequality for $\eta(t)$, as defined in (2.12). We present the argument for the unsymmetrized version; it will be apparent that some of the estimates can be improved in the symmetrized case. We need only prove the theorem for sufficiently large $p$ : Since the estimates are in a fixed bounded set, the $L^{p}$ estimates imply $L^{p^{\prime}}$ estimates for $p^{\prime}<p$. We therefore choose fixed values of $\varepsilon$ and $p$ satisfying the conditions of the Stability Lemma.

Let

$$
e_{i}(t)=\tilde{x}_{i}-x_{i}, \quad w_{i}(t)=\tilde{\omega}_{i}-\omega_{i},
$$

so that

$$
\eta(t)=\left|e_{i}\right|_{0, p, h}+\left|w_{i}\right|_{-1, p, h} .
$$

Since $\eta(0)=0$, condition (2.12) holds at least for some time interval, say $0 \leqslant t \leqslant T^{*}$. To assess the growth of $e_{i}$, we write

$$
\begin{aligned}
\dot{e}_{i}(t) & =\tilde{u}_{i}^{h}(t)-u\left(x_{i}, t\right) \\
& =\left[\tilde{u}^{h}\left(\tilde{x}_{i}, t\right)-u^{h}\left(x_{i}, t\right)\right]+\left[u^{h}\left(x_{i}, t\right)-u\left(x_{i}, t\right)\right] .
\end{aligned}
$$

Provided $0 \leqslant t \leqslant T^{*}$, we can apply the stability estimate (S1) to the first term and the consistency estimate $(\mathrm{C} 1)$ to the second to obtain

$$
\left|\dot{e}_{i}(t)\right|_{0, p, h} \leqslant C\left(\eta(t)+\delta^{m}+\delta(h / \delta)^{l}\right) .
$$

(Estimate (C4) could be used here to improve the last term.) In an entirely similar manner, we have

$$
\begin{aligned}
\dot{w}_{i}(t)= & \nabla \tilde{u}_{i}^{h} \tilde{\omega}_{i}-\nabla u\left(x_{i}, t\right) \omega\left(x_{i}, t\right) \\
= & {\left[\nabla \tilde{u}^{h}\left(\tilde{x}_{i}, t\right) \tilde{\omega}_{i}-\nabla u^{h}\left(x_{i}, t\right) \omega_{i}\right] } \\
& +\left[\nabla u^{h}\left(x_{i}, t\right)-\nabla u\left(x_{i}, t\right)\right] \omega\left(x_{i}, t\right)
\end{aligned}
$$

and, therefore, by (S2) and (C2),

$$
\left|\dot{w}_{i}(t)\right|_{-1, p, h} \leqslant C\left(\eta(t)+\delta^{m}+(h / \delta)^{l}\right) .
$$

We now use the relation $\delta=c_{0} h^{q}$ to write $(h / \delta)^{l}=C h^{l(1-q)}$. We choose $l$ large enough so that $(h / \delta)^{l} \leqslant C h^{m q}$, viz., $l \geqslant m q /(1-q)$. Combining the two inequalities, we then have

$$
\left|\dot{e}_{i}(t)\right|_{0, p, h}+\left|\dot{w}_{i}(t)\right|_{-1, p, h} \leqslant C_{0}\left(\left|e_{i}\right|_{0, p, h}+\left|w_{i}\right|_{-1, p, h}+h^{m q}\right)
$$

for $0 \leqslant t \leqslant T^{*}$, with initial condition

$$
e_{i}(0)=0, \quad w_{i}(0)=0 .
$$

It follows from standard arguments for differential inequalities that

$$
\left|e_{i}(t)\right|_{0, p, h}+\left|w_{i}(t)\right|_{-1, p, h} \leqslant y(t), \quad 0 \leqslant t \leqslant T^{*},
$$

where $y$ is the solution of $y^{\prime}=C_{0}\left(y+h^{m q}\right), y(0)=0$. Therefore,

$$
\left|e_{i}\right|_{0, p, h}+\left|w_{i}\right|_{-1, p, h} \leqslant C_{1} h^{m q}
$$


as long as (2.12) holds. Here $C_{1}$ depends on $C_{0}$ and $T$ but not on $T^{*}$. Now for $h$ sufficiently small, say $h \leqslant h_{0}$, we have $C_{1} h^{\varepsilon}<\frac{1}{2}$, so that

$$
C_{1} h^{m q} \leqslant h^{m q-\varepsilon} / 2 \text {. }
$$

Thus for $h \leqslant h_{0}, \eta(t)$ is bounded by $h^{m q-\varepsilon} / 2$ until it reaches $h^{m q-\varepsilon}$. This means it can never reach $h^{m q-\varepsilon}$, so that (3.1) is true for all $0 \leqslant t \leqslant T$. We have now proved (1) and (3) of the Convergence Theorem.

The remaining statements are a consequence of (3.1) and the stability and consistency estimates. For (2) we write

$$
\tilde{u}_{i}^{h}(t)-u\left(x_{i}, t\right)=\left[\tilde{u}_{i}^{h}(t)-u_{i}^{h}(t)\right]+\left[u^{h}\left(x_{i}, t\right)-u\left(x_{i}, t\right)\right] .
$$

Applying (S1) and (C1) again, we obtain the stated estimate. Similarly,

$$
\tilde{u}^{h}(x, t)-u(x, t)=\left[\tilde{u}^{h}(x, t)-u^{h}(x, t)\right]+\left[u^{h}(x, t)-u(x, t)\right],
$$

and (4) follows from (S3) and (C1). In exactly the same way, (S4) and (C3) imply (5). This completes the proof of the theorem.

We now discuss the consistency estimates. The consistency error separates naturally into two parts, the error due to the smoothing by $\varphi_{\delta}$ and the error of discretization. The behavior of the first part is summarized in the next lemma.

LEMMA 3.1. The following estimates hold uniformly in $x$ and $t, 0 \leqslant t \leqslant T$ :

(1) $\left|\left(\omega * \varphi_{\delta}\right)(x, t)-\omega(x, t)\right| \leqslant C \delta^{m}$,

(2) $\left|\left(K_{\delta} * \omega\right)(x, t)-u(x, t)\right| \leqslant C \delta^{m}$,

(3) $\left|\nabla\left(K_{\delta} * \omega\right)(x, t)-\nabla u(x, t)\right| \leqslant C \delta^{m}$.

Here $*$ denotes convolution. Since $u=K * \omega$, each of the three quantities is the difference between a function and its convolution with $\varphi_{\delta}$. Such estimates can be derived in either of two standard ways, by expanding the function in a Taylor series (see [19] or [26]), or by using the Fourier transform and observing that $\hat{\varphi}_{\delta}(\xi)-1=$ $\mathrm{O}\left(\delta^{m}\right)$ (see [I] or [1]). The latter argument applies under the current assumptions on $\varphi$.

It was shown by Cottet and Raviart [14], [15], [26] that the discretization error could be treated directly as a quadrature error in Lagrangian coordinates. The basic quadrature rule needed here is the following.

LEMma 3.2. Let $F$ be a function on $\mathbf{R}^{n}$ such that $D^{\beta} F \in L^{1}\left(\mathbf{R}^{n}\right)$ for $|\beta| \leqslant l$, where $l \geqslant n+1$. Then,

$$
\left|\sum_{j \in \Lambda} F(j h) h^{n}-\int F(x) d x\right| \leqslant C_{l} h^{l} \sum_{|\beta|=l}\left|D^{\beta} F\right|_{L^{1}} .
$$

This lemma was proved by Cottet and Raviart using the Bramble-Hilbert Lemma. Another proof, based on the Poisson Summation Formula, was given by Greengard in [1]. For the sake of completeness we recall the essence of the latter proof here: According to the Poisson formula

$$
\sum_{j} F(j h) h^{n}-\int F(x) d x=\sum_{k \neq 0} \hat{F}\left(\frac{2 \pi k}{h}\right) .
$$

Since $D^{\beta} F \in L^{1}$, we have

$$
\left|\xi^{\beta} \hat{F}(\xi)\right| \leqslant\left|D^{\beta} F\right|_{L^{1}}
$$


For given $\xi$, we can choose $\beta$ so that $\left|\xi^{\beta}\right| \geqslant|\xi|^{l} / n^{l / 2}$. Therefore,

$$
\left|\sum_{k \neq 0} \hat{F}\left(\frac{2 \pi k}{h}\right)\right| \leqslant\left(\frac{h \sqrt{n}}{2 \pi}\right)^{l}\|F\| \sum_{k \neq 0}|k|^{-l},
$$

where $\|F\|$ is the sum over $\beta$ of the $L^{1}$-norms. The sum on the right converges provided $l \geqslant n+1$. More explicit estimates can be given for the constants; see [1].

In order to apply this lemma, we need the following bounds for $K_{\delta}$ and its derivatives on $L^{1}$ :

LEMMA 3.3. For arbitrary $R>0$ we have

$$
\int_{|x| \leqslant R}\left|D^{\beta} K_{\delta}(x)\right| d x \leqslant \begin{cases}C, & |\beta|=0 \\ C|\log \delta|, & |\beta|=1 \\ C \delta^{1-|\beta|}, & |\beta| \geqslant 2\end{cases}
$$

This integral estimate follows directly from the pointwise estimates

$$
\begin{array}{ll}
\left|D^{\beta} K_{\delta}(x)\right| \leqslant C \delta^{-2-|\beta|}, & |x| \leqslant \delta, \\
\left|D^{\beta} K_{\delta}(x)\right| \leqslant C|x|^{-2-|\beta|}, & |x| \geqslant \delta .
\end{array}
$$

These in turn can be obtained easily from our assumptions (F1)-(F4). (They were proved in more general circumstances by a longer argument in [I].) To derive (3.2) we recall from (F1) that $f(\rho)=\rho^{3} f^{\#}(\rho)$ for a smooth function $f^{\#}$. Substituting in (1.4), we have, with $r=|x|$,

$$
K_{\delta}(x)=-\left(4 \pi \delta^{3}\right)^{-1} f^{\#}(r / \delta) x \times,
$$

from which (3.2) is clear. On the other hand, (F4) shows that

$$
\left|(\partial / \partial r)^{k} f(r / \delta)\right| \leqslant C \delta^{-k}(r / \delta)^{-k}=C r^{-k}
$$

for $k \geqslant 1$ and $r \geqslant \delta$, and (3.3) follows inductively from (1.4).

Consistency estimates such as (C1)-(C3) can be obtained in a straightforward manner, as was noted in [14], [15], by combining the last three lemmas. For (C1) we write

$$
u^{h}(x, t)-u(x, t)=\{1\}+\{2\}
$$

with

$$
\begin{aligned}
& \{1\}=\sum_{j} K_{\delta}\left(x-x_{j}\right) \omega_{j} h^{3}-\int K_{\delta}\left(x-x^{\prime}\right) \omega\left(x^{\prime}\right) d x^{\prime}, \\
& \{2\}=\left(K_{\delta} * \omega\right)(x)-u(x, t) .
\end{aligned}
$$

The second term is $O\left(\delta^{m}\right)$ by Lemma 3.1. To apply Lemma 3.2 to the first term in Lagrangian coordinates, let

$$
F(\alpha)=K_{\delta}\left(x-\Phi^{t}(\alpha)\right) \omega\left(\Phi^{t}(\alpha)\right) .
$$

Since $x_{j}=\Phi^{t}(j h)$ and the flow is volume-preserving,

$$
\{1\}=\sum_{j \in \Lambda} F(j h) h^{3}-\int F(\alpha) d \alpha .
$$


Therefore,

$$
|\{1\}| \leqslant C h^{\prime}\left|D^{\prime} F\right|_{L^{1}}
$$

Finally, since $\Phi^{t}$ and $\omega$ are smooth, Lemma 3.3 implies that

$$
\left|D^{\prime} F\right|_{L^{1}} \leqslant C \delta^{1-l}
$$

and (C1) is established. (C2) and (C3) follow from a similar argument; in fact, (C3) is really a special case of (C2).

4. Some Lemmas. In this section we discuss some preliminaries which are used in the stability argument. They will be needed primarily to estimate integral operators with kernels related to the velocity kernel $K_{\delta}$.

The grid covering the initial vorticity can in principle be extended to a partition of the entire plane. With $j=\left(j_{1}, j_{2}, j_{3}\right)$ a multi-integer, let

$$
Q_{j}=\left\{\alpha \in \mathbf{R}^{3}:-h / 2<\alpha_{i}-j_{i} h<h / 2, i=1,2,3\right\} .
$$

The particle trajectory $x_{j}(t)$ begins at the center of $Q_{j}$. At later time $t$, the cube $Q_{j}$ has evolved to a cell $B_{j}=\Phi^{t}\left(Q_{j}\right)$, and the collection $\left\{B_{j}\right\}$ again partitions $\mathbf{R}^{3}$. Since the flow is incompressible, each $B_{j}$ has volume $h^{3}$, and since the mapping $\Phi^{t}$ has bounded derivatives, the diameters of the cells $B_{j}$ are uniformly of order $h$. We will often use the partition $\left\{B_{j}\right\}$ in estimating integrals.

We first recall a standard fact about integral operators on $L^{p}$ spaces, in both continuous and discrete versions. For the proof, see e.g., [16, Section 0.C].

Lemma 4.1. (a) Suppose $\mathscr{K}\left(x, x^{\prime}\right)$ is a $3 \times 3$ matrix of measurable functions of $\left(x, x^{\prime}\right) \in S_{1} \times S_{2} \subseteq \mathbf{R}^{3} \times \mathbf{R}^{3}$. Define the operator $\mathscr{K}: L^{p}\left(S_{2} ; \mathbf{R}^{3}\right) \rightarrow L^{p}\left(S_{1} ; \mathbf{R}^{3}\right)$ by

$$
(\mathscr{K} f)(x)=\int_{S_{2}} \mathscr{K}\left(x, x^{\prime}\right) f\left(x^{\prime}\right) d x^{\prime}, \quad x \in S_{1} .
$$

Then, we have

$$
|\mathscr{K} f|_{L^{p}\left(S_{1}\right)} \leqslant\|\mathscr{K}\||f|_{L^{p}\left(S_{2}\right)},
$$

where $\|\mathscr{K}\|$ is the smallest number satisfying

$$
\int_{S_{2}}\left|\mathscr{K}\left(y, x^{\prime}\right)\right| d x^{\prime} \leqslant\|\mathscr{K}\|, \quad \int_{S_{1}}\left|\mathscr{K}\left(x, y^{\prime}\right)\right| d x \leqslant\|\mathscr{K}\|,
$$

for all $y \in S_{1}, y^{\prime} \in S_{2}$.

(b) With $S_{1}, S_{2}$ as above, suppose $\mathscr{K}_{i j}$ is a $3 \times 3$ matrix defined for all multiintegers $i, j$ with ih $\in S_{1}$, jh $\in S_{2}$. If $\mathscr{K}: L_{h}\left(S_{2} ; \mathbf{R}^{3}\right) \rightarrow L_{h}\left(S_{1} ; \mathbf{R}^{3}\right)$ is defined by

$$
(\mathscr{K} f)_{i}=\sum_{j h \in S_{2}} \mathscr{K}_{i j} f_{j} h^{3}
$$

then

$$
|\mathscr{K} f|_{L_{h}\left(S_{1}\right)} \leqslant\|\mathscr{K}\||f|_{L R_{h}\left(S_{2}\right)},
$$

where $\|\mathscr{K}\|$ is the smallest number so that

$$
\sum_{l h \in S_{2}}\left|\mathscr{K}_{i l}\right| h^{3} \leqslant\|\mathscr{K}\|, \quad \sum_{l h \in S_{1}}\left|\mathscr{K}_{l j}\right| h^{3} \leqslant\|\mathscr{K}\|
$$

for all ih $\in S_{1}$ and $j h \in S_{2}$. 
In applying Lemma 4.1 we use quantities of the form

$$
M_{i j}^{(l)}=\sup _{\substack{\left|y_{i j}\right| \leqslant C_{0} \delta \\|\beta| \leqslant l}}\left|D^{\beta} K_{\delta}\left(x_{i}-x_{j}+y_{i j}\right)\right|
$$

for some constant $C_{0}$, estimating uniformly over $B_{j}$ or $B_{i}$. Here, $x_{i}=x_{i}(t)=\Phi^{t}(i h)$ for some fixed time $t$. We then have bounds for integral operators in terms of the following discretized $L^{1}$-norms of $D^{\beta} K_{\delta}$.

LEMMA 4.2. With $x_{j}=x_{j}(t)$, we have

$$
\sum_{|j h|<R} M_{i j}^{(l)} h^{3} \leqslant \begin{cases}C, & l=0, \\ C|\log \delta|, & l=1, \\ C \delta^{-l+1}, & l \geqslant 2,\end{cases}
$$

for all $i$ with $|i h|<R$. Here, $C$ depends only on $R, C_{0}$, and bounds for the flow.

This is a restatement of Lemma 3.2 in [I]. The proof, in Section 5 of [I], depends on the pointwise estimates (3.2), (3.3). They were derived in Lemma 5.1 of [I] in a more general setting.

Because we measure the vorticity in $W^{-1, p}$, we will need a version of Lemma 4.1 for operators from $W^{-1, p}$ to $L^{p}$ or $W_{h}^{-1, p}$ to $L_{h}$. This is the purpose of the next lemma.

LEMMA 4.3. (a) With notation as in Lemma 4.1(a), suppose $S_{2}=\left\{z^{\prime}:\left|z^{\prime}\right|<R+1\right\}$. For $f \in W^{-1, p}\left(\mathbf{R}^{3}\right)$ with $\operatorname{supp} f \subseteq\left\{\left|z^{\prime}\right|<R\right\}$, we have

$$
|\mathscr{K} f|_{0, p} \leqslant C\left(\|\mathscr{K}\|+\left\|D_{z^{\prime}} \mathscr{K}\right\|\right)|f|_{-1, p}
$$

with operator norm as in (4.2).

(b) With notation as in Lemma 4.1(b), suppose $S_{2}$ is as above. For $f \in W_{h}^{-1, p}$ with $\operatorname{supp} f \subseteq\left\{\left|z^{\prime}\right|<R\right\}$, we have

$$
|\mathscr{K} f|_{0, p, h} \leqslant C\left(\|\mathscr{K}\|+\left\|D_{j}^{h} \mathscr{K}\right\|\right)|f|_{-1, p, h},
$$

where $D_{j}^{h}$ is the forward-difference quotient with respect to $j$ and the norms are as in (4.4).

A similar statement holds for operators from discrete to continuous spaces with obvious modifications. The lemma is easily proved by dualizing a corresponding statement for operators from $L^{p}$ to $W^{1, p}$. See the proof of Lemma 3.4 in [I].

Finally, it will be important to use the mapping properties of $K_{\delta}$ on $L^{p}$ spaces. As a consequence of the Calderon-Zygmund inequality we know that the first derivative of $K_{\delta}$ is a bounded operator on $L^{p}$ :

LEMMA 4.4. For $f \in L^{p}\left(\mathbf{R}^{3} ; \mathbf{R}^{3}\right)$ of bounded support, $1<p<\infty$, we have

$$
\left|\left(D K_{\delta}\right) * f\right|_{L^{p}} \leqslant C|f|_{L^{p}}
$$

where $D$ denotes any first derivative.

Proof. From the representation (1.1), (1.2) we have for $f \in L^{2} \cap L^{\infty}$,

$$
K * f=-\nabla \times\left(\nabla^{2}\right)^{-1} f
$$


so that

$$
K_{\delta} * f=\left(K * \varphi_{\delta}\right) * f=K *\left(\varphi_{\delta} * f\right)
$$

and

$$
D K_{\delta} * f=-D \cdot \nabla \times\left(\nabla^{2}\right)^{-1}\left(\varphi_{\delta} * f\right) .
$$

The second derivatives of $\left(\nabla^{2}\right)^{-1}$ are integral operators with average value zero, plus delta functions for the repeated derivatives. It follows from the Calderon-Zygmund inequality (e.g., see [30]) that

$$
\left|D K_{\delta} * f\right|_{L^{p}} \leqslant C\left|\varphi_{\delta} * f\right|_{L^{p}}
$$

Finally, $\left|\varphi_{\delta} * f\right|_{L^{p}} \leqslant C|f|_{L^{p}}$ since the $L^{1}$-norm of $\varphi_{\delta}$ is independent of $\delta$.

5. Proof of the Stability Lemma. To begin the stability argument we recall the assumptions on the parameters,

$$
\begin{gathered}
3 q>1+2 \varepsilon \\
0<\varepsilon<q / 2, \\
p>3 / \varepsilon .
\end{gathered}
$$

For simplicity, we will take the constant $c_{0}=\delta / h^{q}$ to be 1 . With $e_{j}=\tilde{x}_{j}-x_{j}$ and $w_{j}=\tilde{\omega}_{j}-\omega_{j}$ as before, the assumed inequality (2.12) can be written as

$$
\eta \equiv\left|e_{j}\right|_{0, p, h}+\left|w_{j}\right|_{-1, p, h} \leqslant \delta^{4} h^{-\varepsilon}
$$

since we suppose that $m \geqslant 4$. Below we will need maximum estimates for $e_{j}$ and $w_{j}$ which are consequences of this bound. From the obvious inequality

$$
\max _{j}\left|e_{j}\right|^{p} h^{3} \leqslant \sum_{j}\left|e_{j}\right|^{p} h^{3}
$$

we find

$$
\max \left|e_{j}\right| \leqslant h^{-3 / p}\left|e_{j}\right|_{0, p, h}
$$

and combining (5.3) with (5.4) we have

$$
\max \left|e_{j}\right| \leqslant h^{-2 \varepsilon} \delta^{4} \text {. }
$$

In a similar way we can use (2.10) to obtain

$$
\max \left|w_{j}\right| \leqslant 2 h^{-1-2 \varepsilon} \delta^{4} \text {. }
$$

We first verify (S1). As in [I], we write $\tilde{u}_{i}^{h}-u_{i}^{h}$ symbolically as

$$
\begin{aligned}
\tilde{u}_{i}^{h}-u_{i}^{h} & =\tilde{K}_{\delta} \tilde{\omega}-K_{\delta} \omega \\
& =\left(\tilde{K}_{\delta}-K_{\delta}\right) \omega+K_{\delta} w+\left(\tilde{K}_{\delta}-K_{\delta}\right) w \\
& \equiv v_{i}^{(1)}+v_{i}^{(2)}+v_{i}^{(3)},
\end{aligned}
$$

where $\tilde{K}_{\delta}$ is evaluated at $\tilde{x}_{i}-\tilde{x}_{j}$, etc. Condition (5.5) implies, in particular, that $\max \left|e_{j}\right| \leqslant \delta$. Under this latter assumption, we have

$$
\left|v_{i}^{(1)}\right|_{0, p, h} \leqslant C\left|e_{j}\right|_{0, p, h} .
$$

This can be shown just as in [I, pp. 16-17], for the special case $p=2$, taking into account the present Lemmas 4.1, 4.2, 4.4 and 3.3; the argument will not be repeated here. (For the extension to $L^{p}$ in two dimensions, see [II, pp. 47-49].) 
Next, we estimate

$$
v_{i}^{(2)}=\sum_{j} K_{\delta}\left(x_{i}-x_{j}\right) w_{j} h^{3} .
$$

It is convenient to dualize; for $g_{i} \in L_{h}^{p^{*}}, g_{i}$ with support in a fixed bounded set containing the support of the vorticity, we write

$$
\left(v_{i}^{(2)}, g_{i}\right)_{h}=\sum_{i j} K_{\delta}\left(x_{i}-x_{j}\right) w_{j} g_{i} h^{6}=\left(w_{j}, b_{j}\right)_{h},
$$

where

$$
b_{j}=\sum_{i} K_{\delta}\left(x_{i}-x_{j}\right) g_{i} h^{3}
$$

We will show that

$$
\left|b_{j}\right|_{1, p^{*}, h} \leqslant C\left|g_{i}\right|_{0, p^{*}, h}
$$

it then follows that

$$
\left|\left(v_{i}^{(2)}, g_{i}\right)_{h}\right| \leqslant C\left|w_{j}\right|_{-1, p, h}\left|g_{i}\right|_{0, p^{*}, h},
$$

and since $g$ is arbitrary,

$$
\left|v_{i}^{(2)}\right|_{0, p, h} \leqslant C\left|w_{j}\right|_{-1, p, h} \text {. }
$$

To verify (5.9), we concentrate on estimating $\left|D_{j}^{h} b_{j}\right|_{0, p^{*}, h}$; the term $\left|b_{j}\right|_{0, p^{*}, h}$ can be handled more easily. We have

$$
D_{j}^{h} b_{j}=\sum_{i} D_{j}^{h} K_{\delta}\left(x_{i}-x_{j}\right) g_{i} h^{3} .
$$

We treat this expression in a manner similar to the $v^{(11)}$-term in [I]. Let $\gamma(x)$ be the step function defined by $\gamma(x)=g_{i}$ for $x \in B_{i}$, and let $\beta\left(x^{\prime}\right)=D_{j}^{h} b_{j}$ for $x^{\prime} \in B_{j}$. Also, let

$$
\mathscr{K}\left(x, x^{\prime}\right)=D_{j}^{h} K_{\delta}\left(x_{i}-x_{j}\right), \quad x \in B_{i}, x^{\prime} \in B_{j} .
$$

Then (5.11) is equivalent to

$$
\beta\left(x^{\prime}\right)=\int \mathscr{K}\left(x, x^{\prime}\right) \gamma(x) d x,
$$

and because the flow is volume-preserving,

$$
|\beta|_{0, p^{*}}=\left|D_{j}^{h} b_{j}\right|_{0, p^{*}, h}, \quad|\gamma|_{0, p^{*}}=\left|g_{i}\right|_{0, p^{*}, h} .
$$

It will therefore be enough to show that

$$
|\beta|_{0, p^{*}} \leqslant C|\gamma|_{0, p^{*}}
$$

Since $D_{j}^{h}$ is a first-order difference operator, we have

$$
D_{j}^{h} K_{\delta}\left(x_{i}-x_{j}\right)=D_{j} K_{\delta}\left(x_{i}-x_{j}\right)+r_{i j} \text {, }
$$

with $\left|r_{i j}\right| \leqslant C M_{i j}^{(2)} h$. Also, for $x \in B_{i}, x^{\prime} \in B_{j}$, the Mean Value Theorem implies

$$
\left|D K_{\delta}\left(x-x^{\prime}\right)-D K_{\delta}\left(x_{i}-x_{j}\right)\right| \leqslant C M_{i j}^{(2)} h .
$$

Thus, we can write

$$
\mathscr{K}\left(x, x^{\prime}\right) \equiv \mathscr{K}_{1}\left(x, x^{\prime}\right)+\mathscr{K}_{2}\left(x, x^{\prime}\right)
$$


with

$$
\mathscr{K}_{1}\left(x, x^{\prime}\right)=D K_{\delta}\left(x-x^{\prime}\right)
$$

and

$$
\left|\mathscr{K}_{2}\left(x, x^{\prime}\right)\right| \leqslant C M_{i j}^{(2)} h, \quad x \in B_{i}, x^{\prime} \in B_{j} .
$$

The $\mathscr{K}_{1}$-term in $\beta$ is just $\left(D K_{\delta} * \gamma\right)\left(x^{\prime}\right)$, and according to Lemma 4.4,

$$
\left|\mathscr{K}_{1} \gamma\right|_{0, p^{*}} \leqslant C|\gamma|_{0, p^{*}}
$$

On the other hand, the $\mathscr{K}_{2}$-term can be estimated, using Lemmas 4.1(a) and 4.2, by

$$
\left|\mathscr{K}_{2} \gamma\right|_{0, p^{*}} \leqslant C \delta^{-1} h|\gamma|_{0, p^{*}} \leqslant C|\gamma|_{0, p^{*}}
$$

It remains to estimate

$$
v_{i}^{(3)}=\sum_{j}\left[K_{\delta}\left(\tilde{x}_{i}-\tilde{x}_{j}\right)-K_{\delta}\left(x_{i}-x_{j}\right)\right] w_{j} h^{3} .
$$

We can substitute

$$
K_{\delta}\left(\tilde{x}_{i}-\tilde{x}_{j}\right)-K_{\delta}\left(x_{i}-x_{j}\right)=\mathscr{K}_{i j} \cdot\left(e_{i}-e_{j}\right)
$$

with

$$
\mathscr{K}_{i j}=\int_{0}^{1} \nabla K_{\delta}\left(x_{i}-x_{j}+s\left(e_{i}-e_{j}\right)\right) d s,
$$

so that $\left|\mathscr{K}_{i j}\right| \leqslant C M_{i j}^{(1)}$. Using the above, we write

$$
v_{i}^{(3)}=\sum_{j} \mathscr{K}_{i j} e_{i} w_{j} h^{3}-\sum_{j} \mathscr{K}_{i j} e_{j} w_{j} h^{3} \equiv v_{i}^{(31)}-v_{i}^{(32)} .
$$

For the first term, $e_{i}$ factors out of the sum, and we find

$$
\begin{aligned}
\left|v_{i}^{(31)}\right|_{0, h} & \leqslant C\left|e_{i}\right|_{0, h} \cdot \max _{i}\left|\sum_{j} \mathscr{K}_{i j} w_{j} h^{3}\right| \\
& \leqslant C\left|e_{i}\right|_{0, h} \cdot\left|w_{j}\right|_{\max } \cdot \max _{i} \sum_{j} M_{i j}^{(1)} h^{3} \\
& \leqslant C\left|e_{i}\right|_{0, h} \cdot h^{-1-2 \varepsilon} \delta^{4} \cdot|\log \delta|
\end{aligned}
$$

using Lemma 4.2 and (5.6). (Here and below, the norms are understood to be based on $L^{p}$ unless otherwise indicated.) For the remaining term we estimate

$$
\left|v_{i}^{(32)}\right|_{0, h} \leqslant C|\log \delta|\left|e_{j} w_{j}\right|_{0, h},
$$

again by Lemmas 4.1(b) and 4.2, and

$$
\left|e_{j} w_{j}\right|_{0, h} \leqslant\left(\max \left|w_{j}\right|\right)\left|e_{j}\right|_{0, h} \leqslant 2 \delta\left|e_{j}\right|_{0, h}
$$

by (5.6), (5.1). Combining the last three inequalities, we have

$$
\left|v_{i}^{(3)}\right|_{0, h} \leqslant C\left(\left|e_{j}\right|_{0, h}+\left|w_{j}\right|_{-1, h}\right) \text {, }
$$

and this completes the verification of (S1).

The estimate (S3) is obtained in an entirely similar way. We can write the difference

$$
\tilde{u}^{h}-u^{h}=\sum_{j} K_{\delta}\left(x-\tilde{x}_{j}\right) \tilde{\omega}_{j} h^{3}-\sum_{j} K_{\delta}\left(x-x_{j}\right) \omega_{j} h^{3}
$$


as a sum $v^{(1)}+v^{(2)}+v^{(3)}$ as before, except that there is no analogue of the distinction between $\tilde{x}_{i}$ and $x_{i}$. The slight modification of the $v^{(1)}$-term is discussed in [I, p. 20]. The $v^{(2)}$-term is handled as above and is in fact somewhat simpler; the test function $g$ is now a function of the continuous variable $x$, but the treatment of the $j$-difference is unchanged. The term $v^{(3)}$ is handled like $v_{i}^{(32)}$ above with an obvious modification of Lemma 4.1(a) for sums defining a function of a continuous variable.

To establish (S2), we estimate the difference $\tilde{s}_{i}-s_{i}$, where

$$
\tilde{s}_{i}=\sum_{j} \nabla K_{\delta}\left(\tilde{x}_{i}-\tilde{x}_{j}\right) \tilde{\omega}_{j} \tilde{\omega}_{i} h^{3}
$$

and similarly for $s_{i}$. We classify the terms as in the velocity case: In shortened notation,

$$
\begin{aligned}
\tilde{s}_{i}-s_{i} & =(\tilde{K}-K) \omega \omega+K(\tilde{\omega} \tilde{\omega}-\omega \omega)+(\tilde{K}-K)(\tilde{\omega} \tilde{\omega}-\omega \omega) \\
& \equiv s_{i}^{(1)}+s_{i}^{(2)}+s_{i}^{(3)} .
\end{aligned}
$$

We expand $s_{i}^{(1)}$ further as $s_{i}^{(11)}+s_{i}^{(12)}$ with

$$
\begin{aligned}
& s_{i}^{(11)}=\sum_{j}\left[\nabla K_{\delta}\left(x_{i}-\tilde{x}_{j}\right)-\nabla K_{\delta}\left(x_{i}-x_{j}\right)\right] \omega_{j} \omega_{i} h^{3}, \\
& s_{i}^{(12)}=\sum_{j}\left[\nabla K_{\delta}\left(\tilde{x}_{i}-\tilde{x}_{j}\right)-\nabla K_{\delta}\left(x_{i}-\tilde{x}_{j}\right)\right] \omega_{j} \omega_{i} h^{3}
\end{aligned}
$$

and begin with $s_{i}^{(11)}$. Using the Mean Value Theorem we have

$$
s_{i}^{(11)}=\sum_{j}\left[D^{2} K_{\delta}\left(x_{i}-x_{j}+y_{i j}\right)\right] e_{j} \omega_{j} h^{3} \cdot \omega_{i}
$$

with $\left|y_{i j}\right| \leqslant h^{-2 \varepsilon} \delta^{4}$, since the same is true for $e_{j}$ by (5.5). If we remove the $y$ 's in this expression, we have

$$
s_{i}^{(11)}=\sum_{j}\left[D^{2} K_{\delta}\left(x_{i}-x_{j}\right)\right] e_{j} \omega_{j} h^{3} \cdot \omega_{i}+\varepsilon_{i}
$$

with

$$
\left|\varepsilon_{i}\right| \leqslant \sum_{j} C M_{i j}^{(3)} \cdot \delta^{4} h^{-2 \varepsilon}\left|e_{j}\right| h^{3},
$$

so that by Lemmas $4.1,4.2$,

$$
\left|\varepsilon_{i}\right|_{0, h} \leqslant C \delta^{-2} \cdot \delta^{4} h^{-2 \varepsilon}\left|e_{j}\right|_{0, h} \leqslant C^{\prime}\left|e_{j}\right|_{0, h} .
$$

To estimate the sum remaining in $W_{h}^{-1, p}$ we replace one $x$-derivative with a Lagrangian derivative:

$$
D^{2} K_{\delta}\left(x_{i}-x_{j}\right)=\left.\left(d \Phi^{t}\right)^{-1}\left(x_{i}\right) \cdot D_{\alpha}\left\{\nabla K_{\delta}\left(\Phi^{t}(\alpha)-x_{j}\right)\right\}\right|_{\alpha=\alpha_{i}} .
$$

The sum in (5.13) is then a smooth function times

$$
\sum_{j} D_{\alpha} \nabla K_{\delta}\left(\Phi^{t}\left(\alpha_{i}\right)-x_{j}\right) e_{j} \omega_{j} h^{3}
$$

and it suffices to estimate the latter. We now choose a high-order difference operator $D_{\alpha}^{h}$ so that

$$
\left|\left(D_{\alpha}^{h}-D_{\alpha}\right) f\right| \leqslant C h^{r} \sup \left|D^{r+1} f\right|
$$


If we replace $D_{\alpha}$ above by $D_{\alpha}^{h}$, we can estimate the difference in $L_{h}$, and therefore, in $W_{h}^{-1, p}$, by

$$
\left|\sum_{j} C h^{r} M_{i j}^{(r+2)}\right| e_{j}\left|h^{3}\right|_{0, h} \leqslant C^{\prime} h^{r} \delta^{-(r+1)}\left|e_{j}\right|_{0, h},
$$

which is bounded by $\left|e_{j}\right|_{0, h}$, provided $r \geqslant(r+1) q$ or $r \geqslant q /(1-q)$. We are now left with

$$
D_{\alpha}^{h} \sum_{j} \nabla K_{\delta}\left(\Phi^{t}\left(\alpha_{i}\right)-x_{j}\right) e_{j} \omega_{j} h^{3}
$$

which is estimated in $W_{h}^{-1, p}$ by

$$
\sum_{j} \nabla K_{\delta}\left(x_{i}-x_{j}\right) e_{j} \omega_{j} h^{3}
$$

in $L_{h}$. Finally, this last sum is bounded in $L_{h}^{p}$ by $\left|e_{j}\right|_{0, h}$; this can be shown just as in the treatment of (5.11) above. In summary, we have that

$$
\left|s_{i}^{(11)}\right|_{-1, h} \leqslant C\left|e_{j}\right|_{0, h} \text {. }
$$

To estimate $s_{i}^{(12)}$, we again apply the Mean Value Theorem, obtaining

$$
s_{i}^{(12)}=\sum_{j} D^{2} K_{\delta}\left(x_{i}-x_{j}+y_{i j}\right) \omega_{j} h^{3} \cdot e_{i}
$$

with $\max \left|y_{i j}\right| \leqslant h^{-2 \varepsilon} \delta^{4}$ as before. If we remove $y_{i j}$, the error committed can be estimated in $L_{h}$ by $\left|e_{i}\right|_{0, h}$ times the maximum over $i$ of

$$
\sum_{j} M_{i j}^{(3)} \cdot h^{-2 \varepsilon} \delta^{4} h^{3} \leqslant C h^{-2 \varepsilon} \delta^{2} \leqslant C^{\prime} .
$$

We will now have that $\left|s_{i}^{(12)}\right|_{0, h} \leqslant C\left|e_{i}\right|_{0, h}$, provided we show that

$$
\sum_{j} D^{2} K_{\delta}\left(x_{i}-x_{j}\right) \omega_{j} h^{3}
$$

is bounded uniformly in $i$. To verify this, we replace the sum with the integral

$$
\int D^{2} K_{\delta}\left(x_{i}-x^{\prime}\right) \omega\left(x^{\prime}\right) d x^{\prime}
$$

making an error which, according to Lemma 3.2 applied in Lagrangian coordinates, is bounded by

$$
C h^{\prime}\left|D^{l+2} K_{\delta}\right|_{L^{1}} \leqslant C^{\prime} h^{l} \delta^{-(l+1)} .
$$

We have used Lemma 3.3 in the last step. This quantity is bounded provided we choose $l$ large enough so that $q \leqslant l /(l+1)$. Finally, the integral above can be integrated by parts to give

$$
\int K_{\delta}\left(x-x^{\prime}\right) D^{2} \omega\left(x^{\prime}\right) d x^{\prime}
$$

which is bounded by a constant.

To estimate

$$
s^{(2)}=\sum_{j} D K_{\delta}\left(x_{i}-x_{j}\right)\left[\tilde{\omega}_{j} \tilde{\omega}_{i}-\omega_{j} \omega_{i}\right] h^{3}
$$


we write

$$
\tilde{\omega}_{j} \tilde{\omega}_{i}-\omega_{j} \omega_{i}=w_{j} \omega_{i}+\omega_{j} w_{i}+w_{j} w_{i}
$$

and decompose $s^{(2)}$ as $s^{(21)}+s^{(22)}+s^{(23)}$. Since $\omega_{i}$ is smooth, we may ignore it in estimating $s^{(21)}$. If we write the derivative in Lagrangian form, as in $s^{(11)}$, and ignore the Jacobian, which is another smooth factor, we are reduced to estimating

$$
\sum_{j} D_{\alpha} K_{\delta}\left(\Phi^{t}\left(\alpha_{i}\right)-x_{j}\right) w_{j} h^{3}
$$

in $W^{-1, p}$. Suppose we replaced $D_{\alpha}$ with a difference operator $D_{\alpha}^{h}$; then this expression has the form $D_{\alpha}^{h} v_{i}^{(2)}$ with $v_{i}^{(2)}$ as in (5.8), and

$$
\left|D_{\alpha}^{h} v_{i}^{(2)}\right|_{-1, h} \leqslant C\left|v_{i}^{(2)}\right|_{0, h} \leqslant C^{\prime}\left|w_{j}\right|_{-1, h}
$$

using (5.10). It remains to estimate the error in replacing $D_{\alpha}$ by $D_{\alpha}^{h}$. We will estimate

$$
z_{i}=\sum_{j}\left(D_{\alpha}^{h}-D_{\alpha}\right) K_{\delta}\left(\Phi^{t}\left(\alpha_{i}\right)-x_{j}\right) w_{j} h^{3}
$$

in $L h_{h}^{p}$ in terms of $\left|w_{j}\right|_{-1, h}$, using Lemma 4.3(b). Assume $D_{\alpha}^{h}$ is an $r$ th-order difference approximation; then

$$
\left|D_{(j)}^{h}\left(D_{\alpha}^{h}-D_{\alpha}\right) K_{\delta}\left(\Phi^{t}\left(\alpha_{i}\right)-x_{j}\right)\right| \leqslant C h^{r} M_{i j}^{(r+2)}
$$

and

$$
h^{r} \sum_{j} M_{i j}^{(r+2)} h^{3} \leqslant C^{\prime} h^{r} \delta^{-(r+1)} \leqslant C^{\prime \prime}
$$

for $r \geqslant q /(1-q)$, as in the treatment of $s^{(11)}$ above. It then follows from the Lemma that $\left|z_{i}\right|_{0, h} \leqslant C\left|w_{j}\right|_{-1, h}$.

The next term is

$$
s_{i}^{(22)}=w_{i} \cdot \sum_{j} D K_{\delta}\left(x_{i}-x_{j}\right) \omega_{j} h^{3} .
$$

To estimate this in $W_{h}^{-1, p}$ by $C\left|w_{i}\right|_{-1, h}$, it suffices to show that the sum and its $i$-difference are uniformly bounded with respect to $i$. It is convenient, and sufficient, to replace $x_{i}$ by a continuous variable $x$ and estimate

$$
\sum_{j} D K_{\delta}\left(x-x_{j}\right) \omega_{j} h^{3}
$$

in $C^{1}$. This can be done just as in the treatment of $(5.14)$ in $s^{(12)}$, replacing the sum by an integral and integrating by parts. We are now left with the term

$$
s_{i}^{(23)}=\sum_{j} D K_{\delta}\left(x_{i}-x_{j}\right) w_{i} w_{j} h^{3} .
$$

We will estimate this in $L_{h}^{p}$ by

$$
\max \left|w_{i}\right| \cdot\left|\sum_{j} D K_{\delta}\left(x_{i}-x_{j}\right) w_{j} h^{3}\right|_{0, h} .
$$

We have $\max \left|w_{i}\right| \leqslant 2 h^{-2 \varepsilon-1} \delta^{4}$ from (5.6), and using Lemma 4.3(b) again, as well as Lemma 4.2,

$$
\left|\sum_{j} D K_{\delta}\left(x_{i}-x_{j}\right) w_{j} h^{3}\right|_{0, h} \leqslant C \delta^{-1}\left|w_{j}\right|_{-1, h},
$$


so that

$$
\left|s_{i}^{(23)}\right|_{0, h} \leqslant C h^{-1-2 \varepsilon} \delta^{3}\left|w_{j}\right|_{-1, h} \leqslant C^{\prime}\left|w_{j}\right|_{-1, h}
$$

by (5.1).

The final term is

$$
s_{i}^{(3)}=\sum_{j}\left[D K_{\delta}\left(\tilde{x}_{i}-\tilde{x}_{j}\right)-D K_{\delta}\left(x_{i}-x_{j}\right)\right] r_{i j} h^{3}
$$

with $r_{i j}=\tilde{\omega}_{j} \tilde{\omega}_{i}-\omega_{j} \omega_{i}$. Writing $r_{i j}$ as in (5.15), and using (5.6), we have

$$
\max _{i, j}\left|r_{i j}\right| \leqslant C h^{-1-2 \varepsilon} \delta^{4} \text {. }
$$

By the Mean Value Theorem we can express the sum as

$$
\begin{aligned}
s_{i}^{(3)} & =\sum_{j} D^{2} K_{\delta}\left(x_{i}-x_{j}+y_{i j}\right) r_{i j}\left(-e_{j}+e_{i}\right) h^{3} \\
& \equiv s_{i}^{(31)}+s_{i}^{(32)},
\end{aligned}
$$

with the two terms corresponding to $e_{j}$ and $e_{i}$, respectively. We estimate each in $L{ }_{h}$. Since

$$
\left|s_{i}^{(31)}\right| \leqslant \sum_{j} M_{i j}^{(2)}\left|r_{i j}\right|\left|e_{j}\right| h^{3}
$$

we can apply Lemmas $4.1(\mathrm{~b})$ and 4.2 to obtain

$$
\left|s_{i}^{(31)}\right|_{0, h} \leqslant C h^{-1-2 \varepsilon} \delta^{4} \delta^{-1}\left|e_{j}\right|_{0, h} \leqslant C^{\prime}\left|e_{j}\right|_{0, h} .
$$

Next, we write

$$
s_{i}^{(32)}=e_{i} \cdot \sum_{j} D^{2} K_{\delta}\left(x_{i}-x_{j}+y_{i j}\right) r_{i j} h^{3}
$$

and again use Lemma 4.2 to estimate

$$
\left|\sum_{j} D^{2} K_{\delta}\left(x_{i}-x_{j}+y_{i j}\right) r_{i j} h^{3}\right| \leqslant C h^{-1-2 \varepsilon} \delta^{4} \delta^{-1} \leqslant C^{\prime},
$$

so that $\left|s_{i}^{(32)}\right|_{0, h} \leqslant C^{\prime}\left|e_{j}\right|_{0, h}$. This completes the proof of (S2).

Estimate (S4) is similar to (S3) except for an extra derivative on $K_{\delta}$. We have

$$
\tilde{\omega}^{h}(x)-\omega^{h}(x)=\sum_{j} D K_{\delta}\left(x-\tilde{x}_{j}\right) \tilde{\omega}_{j} h^{3}-\sum_{j} D K_{\delta}\left(x-x_{j}\right) \omega_{j} h^{3},
$$

ignoring indices. We decompose this in three terms, $t^{(1)}+t^{(2)}+t^{(3)}$, as in (S1), and first consider the term

$$
-t^{(1)}(x)=\sum_{j} D^{2} K_{\delta}\left(x-\tilde{x}_{j}+y_{j}(x)\right) e_{j} \omega_{j} h^{3},
$$

with $\left|y_{j}(x)\right| \leqslant h^{-2 \varepsilon} \delta^{4}$. Then,

$$
-t^{(1)}(x)=\int \mathscr{K}\left(x, x^{\prime}\right) f\left(x^{\prime}\right) d x^{\prime},
$$

where

$$
f\left(x^{\prime}\right)=e_{j} \omega_{j}, \quad x^{\prime} \in B_{j},
$$

and

$$
\mathscr{K}\left(x, x^{\prime}\right)=D^{2} K_{\delta}\left(x-x_{j}+y_{j}(x)\right), \quad x^{\prime} \in B_{j} .
$$


Since $|\mathscr{K}| \leqslant M_{i j}^{(2)}$ for $x \in B_{i}$, we have by Lemmas 4.1(a), 4.2,

The term

$$
\left|t^{(1)}\right|_{L^{p}(|x|<R)} \leqslant C \delta^{-1}\left|e_{j}\right|_{0, h} \text {. }
$$

$$
t^{(2)}=\sum_{j} D K_{\delta}\left(x-x_{j}\right) w_{j} h^{3}
$$

can be estimated by duality like the $v^{(2)}$-terms in (S1) or (S3), but more simply. For arbitrary $g(x) \in L^{p}(|x|<R)$ we write

$$
\left(t^{(2)}, g\right)=\sum_{j} \int D K_{\delta}\left(x-x_{j}\right) g(x) d x \cdot w_{j} h^{3} \equiv\left(w_{j}, b_{j}\right)_{h},
$$

where

$$
b_{j}=\int D K_{\delta}\left(x-x_{j}\right) g(x) d x
$$

and establish that

$$
\left|b_{j}\right|_{1, p^{*}, h} \leqslant C \delta^{-1}|g|_{0, p^{*}}
$$

This will then imply as before that

$$
\left|t^{(2)}\right|_{0, p} \leqslant C \delta^{-1}\left|w_{j}\right|_{-1, p, h} .
$$

We verify (5.18) just as in the earlier treatment, except that we estimate directly

$$
\left|D_{j}^{h} D K_{\delta}\left(x-x_{j}\right)\right| \leqslant M_{i j}^{(2)}, \quad x \in B_{i}
$$

without separating the kernel into two different terms. Finally,

$$
t^{(3)}=\sum_{j}\left[D K_{\delta}\left(x-\tilde{x}_{j}\right)-D K_{\delta}\left(x-x_{j}\right)\right] w_{j} h^{3}
$$

can be estimated like $v_{i}^{(3)}$ in (S1), leading to

$$
\left|t^{(3)}\right|_{0, p} \leqslant C\left|e_{j}\right|_{0, p, h}
$$

This concludes the discussion of (S4).

Department of Mathematics

Duke University

Durham, North Carolina 27706

1. C. Anderson \& C. Greengard, “On vortex methods," SiAM J. Numer. Anal., v. 22, 1985, pp. 413-440.

2. C. Anderson, Vortex methods for Flows of Variable Density, Ph. D. Thesis, Univ. of California, Berkeley, 1983.

3. C. R. Anderson, "A vortex method for flows with slight density variation," J. Comput. Phys., v. 61,1985 , pp. $417-444$.

4. J. T. Beale \& A. Majda, "Vortex methods, I: Convergence in three dimensions," Math. Comp., v. 39,1982 , pp. 1-27.

5. J. T. BeAle \& A. MAJda, "Vortex methods, II: Higher order accuracy in two and three dimensions," Math. Comp., v. 39, 1982, pp. 29-52.

6. J. T. Beale \& A. MAJDA, "High order accurate vortex methods with explicit velocity kernels," $J$. Comput. Phys., v. 58, 1985, pp. 188-208.

7. J. T. Beale \& A. MajDa, "Vortex methods for fluid flow in two or three dimensions," Contemp. Math., v. 28, 1984, pp. 221-229.

8. J. T. Beale \& A. MAJDA, "Rates of convergence for viscous splitting of the Navier-Stokes equations," Math. Comp., v. 37, 1981, pp. 243-259. 
9. A. CheER, "Numerical analysis of time dependent flow structure generated by an impulsively started circular cylinder in a slightly viscous incompressible fluid," SIAM J. Sci. Statist. Comput. (To appear.)

10. A. Chorin, "Vortex methods and boundary layer instability," SIAM J. Sci. Statist. Comput., v. 1, 1980, pp. 1-21.

11. A. Chorin, "The evolution of a turbulent vortex”, Comm. Math. Phys., v. 83, 1982, pp. 517-535.

12. A. Chorin \& J. MARSDEN, A Mathematical Introduction to Fluid Mechanics, Springer-Verlag, New York, 1979.

13. A. Chorin, T. Hughes, M. McCracken \& J. Marsden, "Product formulas and numerical algorithms," Comm. Pure Appl. Math., v. 31, 1978, pp. 205-256.

14. G.-H. Cotret \& P.-A. Raviart, "Particle methods for the one-dimensional Vlasov-Poisson equations," SIAM J. Numer. Anal., v. 21, 1984, pp. 52-76.

15. G.-H. CotTet, Méthodes Particulaires pour l'Équation d'Euler dans le Plan. Thèse de $3 \mathrm{e}$ cycle, Université P. et M. Curie, Paris, 1982.

16. G. Folland, Introduction to Partial Differential Equations, Princeton Univ. Press, Princeton, N. J., 1978.

17. C. Greengard, Three-Dimensional Vortex Methods, Ph. D. thesis, Univ. of California, Berkeley, 1984.

18. O. Hald \& V. Del Prete, "Convergence of vortex methods for Euler's equations," Math. Comp., v. 32, 1978, pp. 791-809.

19. O. HALD, “The convergence of vortex methods, II," SIAM J. Numer. Anal., v. 16, 1979, pp. $726-755$.

20. T. Kato, “Nonstationary flows of viscous and ideal fluids in $R^{3}$," J. Funct. Anal., v. 9, 1972, pp. 296-305.

21. A. LeONARD, "Numerical simulation of interacting three-dimensional vortex filaments," Proc. 4th Internat. Conf. Numer. Methods Fluid Dynamics, Lecture Notes in Physics, vol. 35, Springer-Verlag, New York, 1975, pp. 245-249.

22. A. LeONARD, "Vortex methods for flow simulations," J. Comput. Phys., v. 37, 1980, pp. 289-335.

23. A. LeONARD, "Computing three-dimensional incompressible flows with vortex elements," Ann. Rev. Fluid Mech., v. 17, 1985, pp. 523-529.

24. C. Marchioro \& M. Pulvirenti, "Hydrodynamics in two dimensions and vortex theory," Comm. Math. Phys., v. 84, 1982, pp. 483-503.

25. Y. Nakamura, A. LeOnard \& P. Spalart, Numerical Simulation of Vortex Breakdown by the Vortex-Filament Method, AGARD Proceedings \#342, Rotterdam, 1983.

26. P. A. Raviart, An Analysis of Particle Methods, CIME Course, Como, Italy, 1983.

27. P. G. Saffman, "Vortex interactions and coherent structures in turbulence," Transition and Turbulence (R. Meyer, ed.), Academic Press, New York, 1981, pp. 149-166.

28. E. D. Siggia, “Collapse and amplification of a vortex filament," Phys. Fluids, v. 28, 1985, pp. 794-805.

29. P. SPalart, Numerical Simulation of Separated Flows, Ph. D. thesis, Stanford University, 1982.

30. E. M. SteIn, Singular Integrals and Differentiability Properties of Functions, Princeton Univ. Press, Princeton, N. J., 1970.

31. R. TemaM, "Local existence of $C^{\infty}$ solutions of the Euler equations of incompressible perfect fluids," in Turbulence and the Navier-Stokes Equations, Springer-Verlag, New York, 1976, pp. 184-194. 


\title{
Supplement to \\ A Convergent 3-D Vortex Method With Grid-Free Stretching
}

\author{
By J. Thomas Beale
}

In this supplement we discuss the more special consistency estimates (C4)-(C6) arising from the fact that the integral kernels have average value zero. This section is not necessary for the proof of convergence and may be omitted without loss of continuity.

Our strategy will be to rewrite the discretization error before applying the quadrature estimate in such a way that the new integrand has improved $\mathrm{L}^{1}$-estimates. In (C4) we need to compare

$$
v\left(x_{i}\right)=-\frac{1}{4 \pi} \int f(r / \delta) \frac{x_{i}-x^{\prime}}{r^{3}} \times \omega\left(x^{\prime}\right) d x^{\prime}
$$

with the discretization

$$
v_{i}^{h}=-\frac{1}{4 \pi} \sum_{j} f\left(r_{i j} / 8\right) \frac{x_{i}-x_{j}}{r_{i j}^{3}} \times w\left(x_{j}\right) h^{3} ;
$$



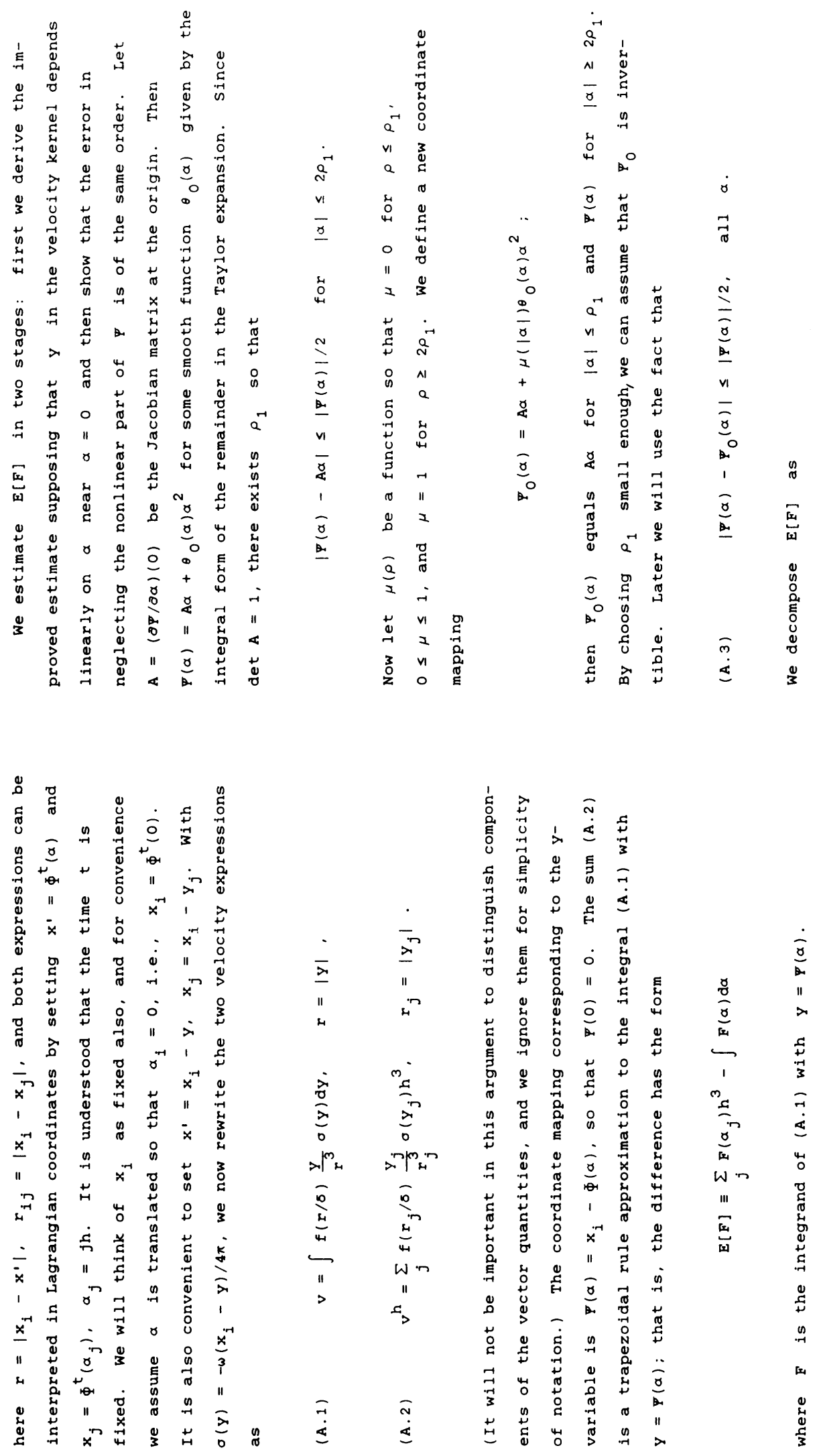

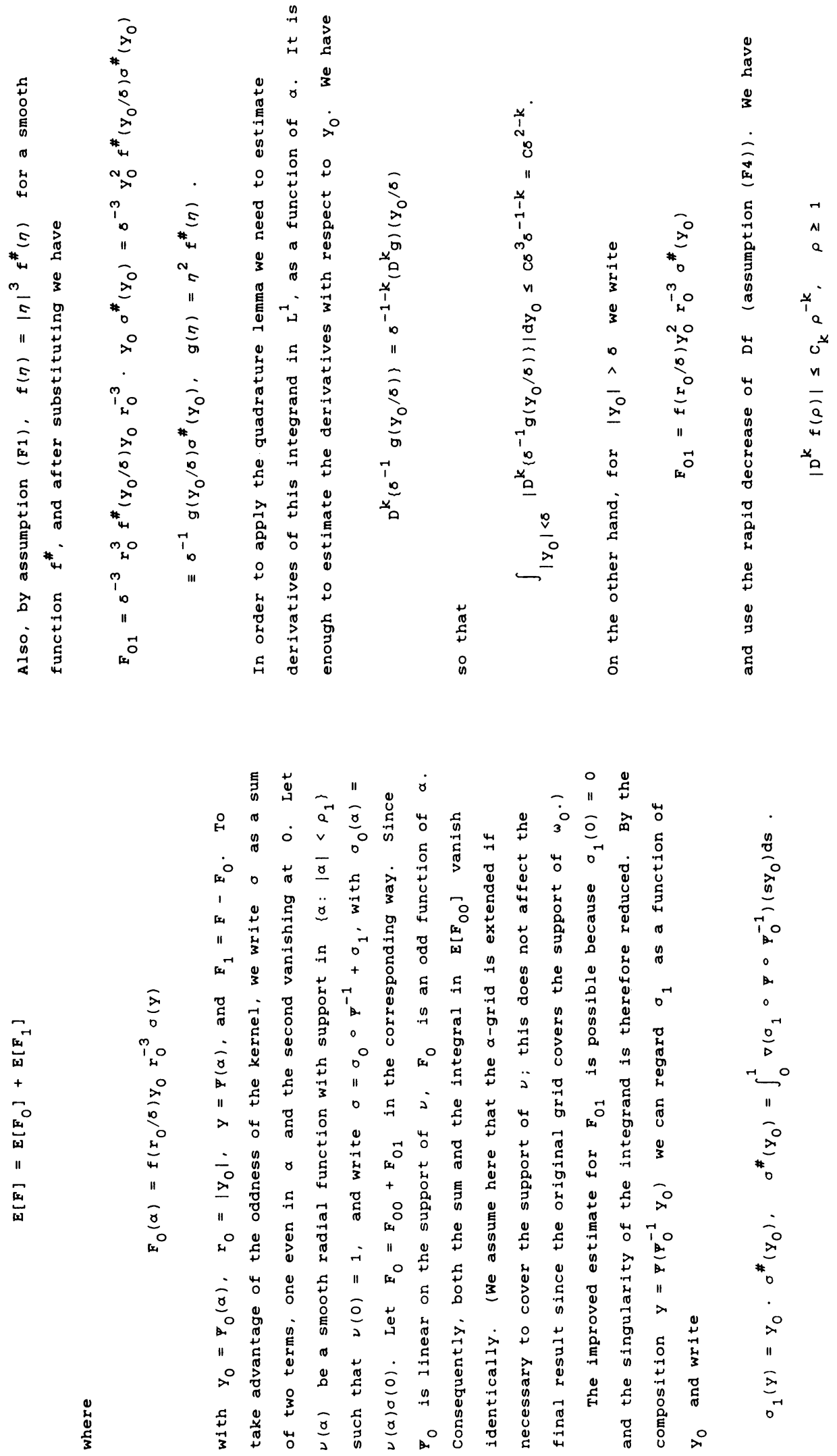

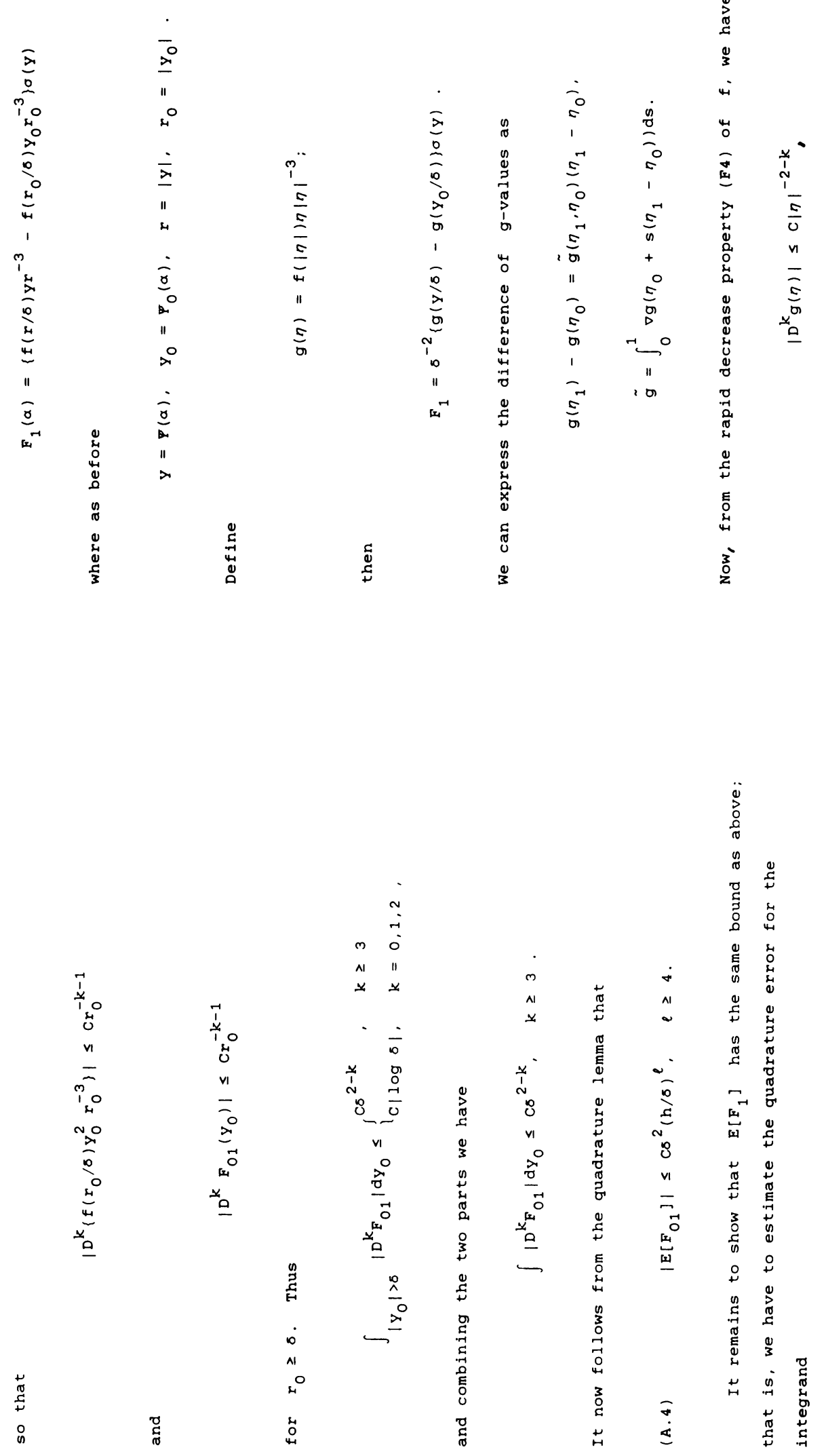

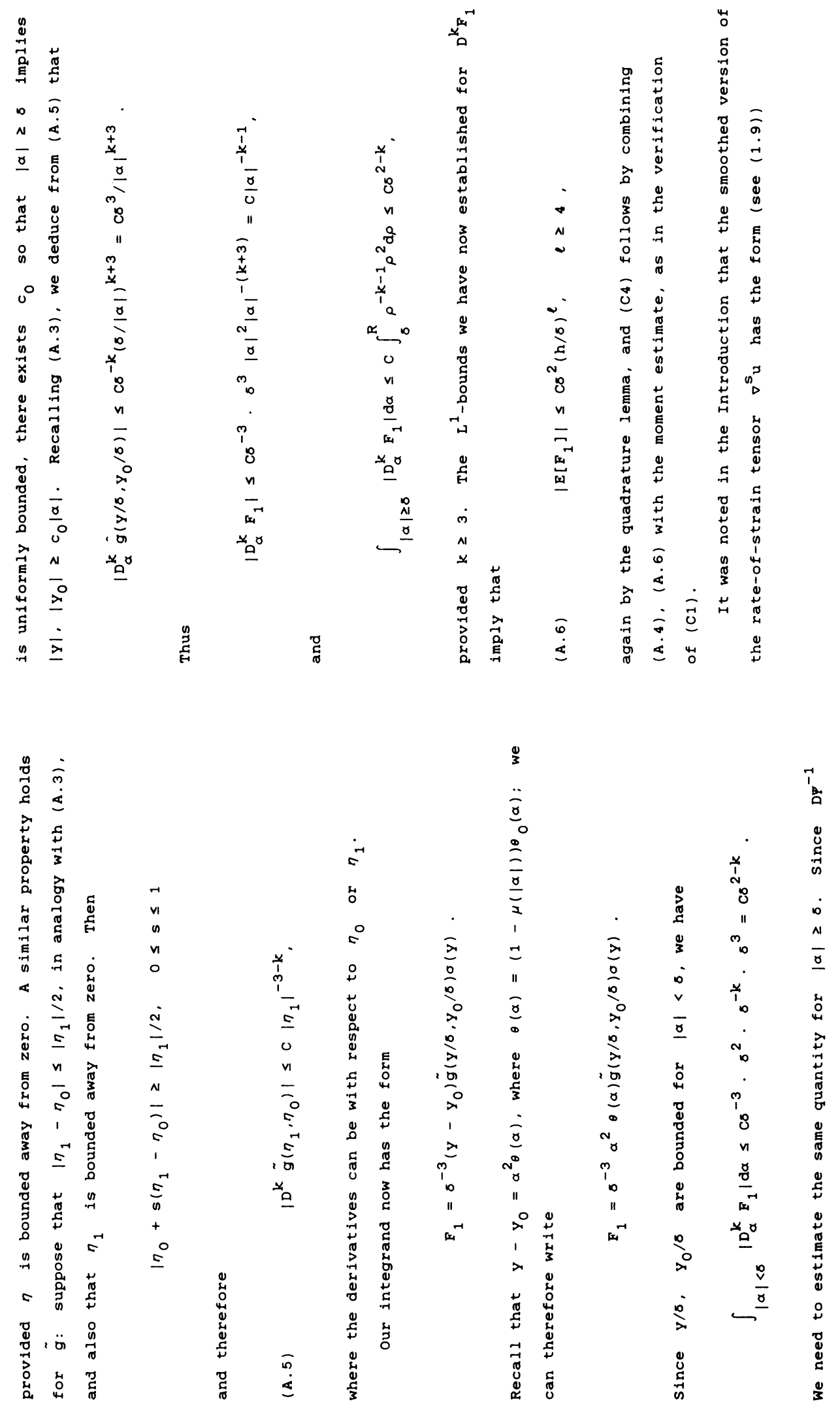

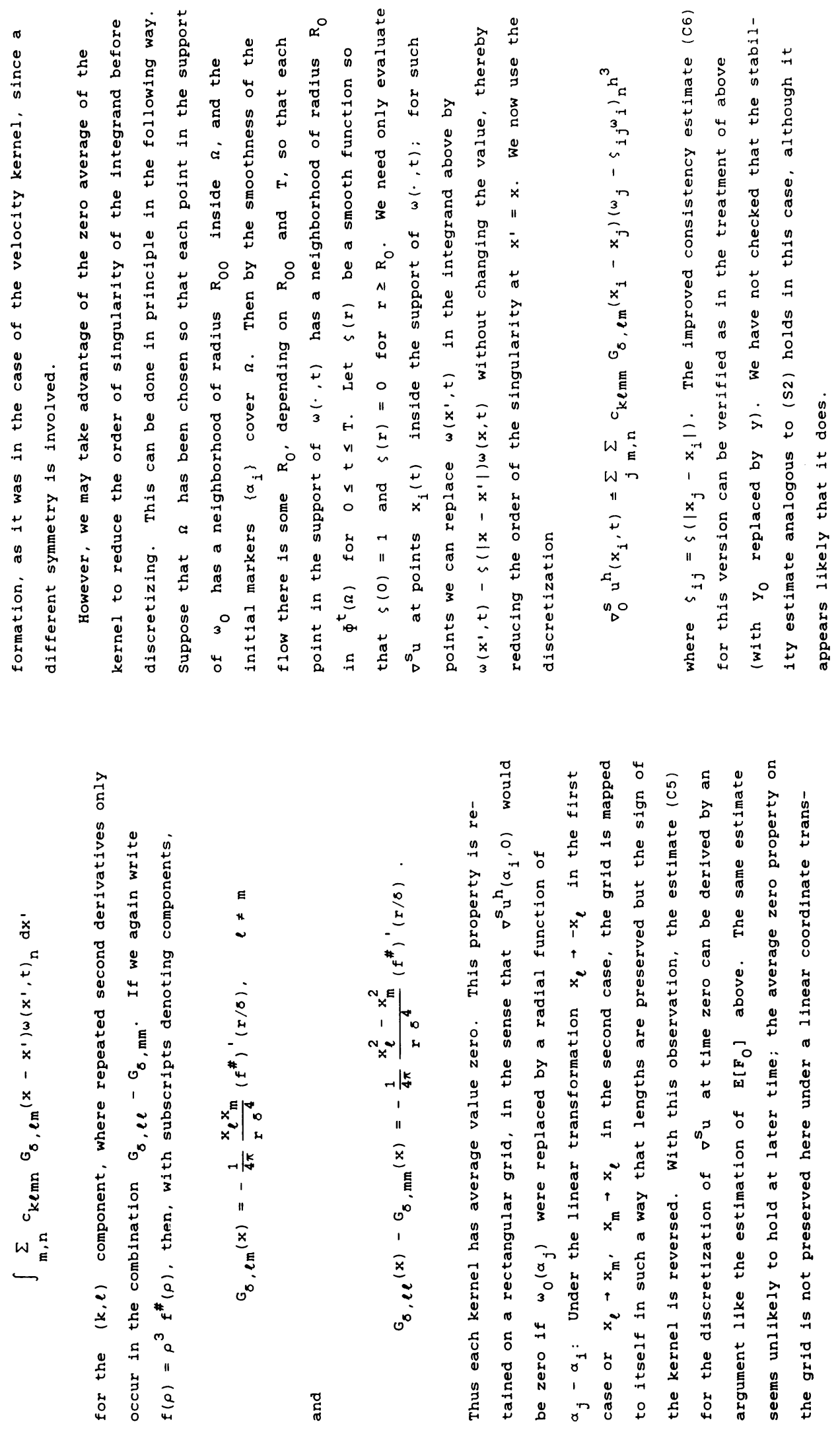